\title{
Design, Molecular Modeling and Synthesis of Some New Purine- diones and Pyridopyrimidine-diones with Anticancer Activity
}

\author{
Ola F. Abou-Ghadir ${ }^{1}$, Alaa M. Hayallah ${ }^{1,2^{*}}$, Samia G. Abdel-Moty ${ }^{1}$, Mostafa A. Hussein ${ }^{1}$, \\ Ahmed S. Aboraia ${ }^{3}$ and Douaa Sayed ${ }^{4}$ \\ ${ }^{1}$ Department of Pharmaceutical Organic Chemistry, Faculty of Pharmacy, Assiut University, Assiut-71526, \\ Egypt. \\ 1olaghadir78@gmail.com, ${ }^{1}$ alaa hayalah@yahoo.com \\ ${ }^{2}$ Department of Pharmaceutical Chemistry, Faculty of Pharmacy Deraya University, El-Minia- Egypt \\ ${ }^{3}$ Department of Medicinal Chemistry, Faculty of Pharmacy, Assiut University, Assiut-71526, Egypt. \\ ${ }^{4}$ Clinical Pathology Department, South Egypt Cancer Institute, Assiut University, Assiut 171515, Egypt \\ ${ }^{*}$ Corresponding author: Alaa Arafat K. M. Hayallah \\ Phone number: Mobile: +201283410110
}

Office: $+2088-2411312$

Fax number: +2088-23332776

Email address: alaa_hayalah@yahoo.com

\begin{abstract}
An olomoucine analogues of 2-[(1-substituted)-2,6-dioxo-2,3,6,7-tetrahydro-1H-purin-8-ylsulfanyl]- $N$-substituted acetamide $\mathbf{6 a - g}$ and $\mathbf{7 a - g}, 1$-substituted-8-[2-(4-substituted phenyl)-2-oxoethylsulphanyl]-3,7-dihydro-1 H-purine-2,6-diones 9a-g and 10a-g, 3-(2-substituted benzyl)-6-(4-substituted phenyl)-1 $H$-thiazolo[2,3-f]purine-2,4-dione 11a-g and 12a-g and their isosteres 3-substituted benzyl-5-methyl-7-substituted-1 $\mathrm{H}$-pyrido[2,3- $d$ ]pyrimidine-2,4-dione 13a-c and 14a-c were designed and synthesized. The target compounds $11 \mathrm{a}-\mathrm{g}$ and $12 \mathrm{a}-\mathrm{g}$ were prepared by cyclodehydration of $9 \mathrm{a}-\mathrm{g}$ and $10 \mathrm{a}-\mathrm{g}$ in PPA, while 13a-c and 14a-c were synthesized by condensation of 6-amino-3-(2-substituted benzyl)-1H-pyrimidine-2,4dione $\mathbf{1 a}$ or $\mathbf{1 b}$ and the appropriate acylacetone in glacial acetic acid. Structures of the new compounds were verified on the basis of their IR, ${ }^{1} \mathrm{H}$ NMR, MS, HRMS and elemental analyses. The newly synthesized compounds were tested for their anticancer activity and most of the tested compounds showed good to excellent inhibition activity against the tested human breast cancer cell line MCF-7 in comparison to doxorubicin as a reference drug.
\end{abstract}

\section{KEYWORDS: olomoucine; cyclin-depenent kinase; synthesis; anticancer INTRODUCTION}

Cancer is a class of diseases characterized by out-of-control cell growth. Cancers can arise from both genetic and lifestyle factors that lead to abnormal regulation in the growth of particular stem cell populations or by the undifferentiation of mature cell types ${ }^{1}$. This aberrant control of cell division can lead to either a benign tumour, which does not spread to other parts of the body and as such is rarely life threatening or to a malignant tumour which can invade other organs, move to other bodily locations (metastasise) and become life threatening ${ }^{1}$. Although the development of novel targeted antitumor drugs have obtained important progress in recent years, cancer remains a major leading cause of death in the world due to drug resistance or undesirable toxic effects ${ }^{2}$. At present, a wide range of cytotoxic drugs with different mechanisms of action are used to treat human cancer, either alone or in combination ${ }^{3}$. In addition, numerous compounds are also in different phases of clinical trials. The main drawback of these cytotoxic drugs is that they do not discriminate between cancerous and normal cell types and are accompanied by toxic side effects that are often cumulative and dose limiting ${ }^{3}$. Despite the enormous progress made in recent years in the understanding of the mechanisms involved in cancer onset and propagation and apart from classical cytotoxic-based therapies, there has been an active search for new approaches, such as protein kinase inhibition ${ }^{4}$.

Cyclin-dependent kinases (cdks) play important roles in cell cycle regulation. Their sequential activation ensures the correct timing and ordering of events required for cell cycle progression ${ }^{5}$. CDKs control the cell division cycle (cdc). These kinases and their regulators are frequently deregulated in human tumours ${ }^{6}$. The purine ring presents in the structure of ATP itself, so it is hardly surprising that amongst the first Cdk inhibitors to be described are 6-dimethylaminopurine and isopentenyladenine which are purine scaffold containing inhibitors ${ }^{4}$. By structural analogy of 6-dimethylaminopurine, which was found to inhibit mitosis of sea urchin embryos without inhibiting protein synthesis, was a substituted purine, olomoucine [2-(2-hydroxyethylamino)-6-benzylamino-9-methyl-purine], chart 1 . Trisubstituted purines in positions C-2, C-6 and N-9 were the most active, and olomoucine was identified as a specific cdk inhibitor which was found to block selectively CDK1, CDK2 and CDK5 kinases at micromolar concentrations ${ }^{4,7}$. In addition, the pyrimidine and purine ring systems undoubtedly belong to the most ubiquitous heterocycles in nature, as they represent the main structure of many biologically significant compounds, including nucleosides and nucleotides. Several of the latter heterocycles possess a multitude of pronounced biological activities ${ }^{8}$. These derivatives have been utilized extensively in medicinal chemistry due to their privileged structure that show various pharmacological activities, such as, anticancer ${ }^{9-15}$, anti-inflammatory ${ }^{16-20}$, anticonvulsant $21-23$ and antibacterial $12,18,24-26$ and antidiabetic activities through inhibition of phosphoenolpyruvate carboxykinase (PEPCK) ${ }^{27,28}$ among others. Moreover, recently 1,8-disubstituted purine-2,6-diones [AH-216 (II), AH-217 (III); chart 2] were reported to possess potent antitumor activity against breast cancer and leukemia ${ }^{10}$. For these reasons, many analogues and derivatives of purine and pyrimidine have been synthesised and developed as pharmacologically active compounds ${ }^{8}$. 
In view of the above-mentioned facts and in continuation of our interest in the synthesis of new heterocyclic compounds comprising in their skeletons the purine-2,6-dione moieties and screening of their biological activities, herein we report the synthesis and anticancer activity of new purine-diones and their isosters pyridopyrimidine diones.

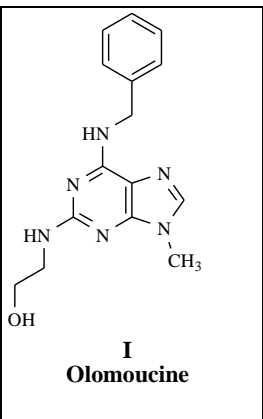

Chart 1; structure of olomoucine

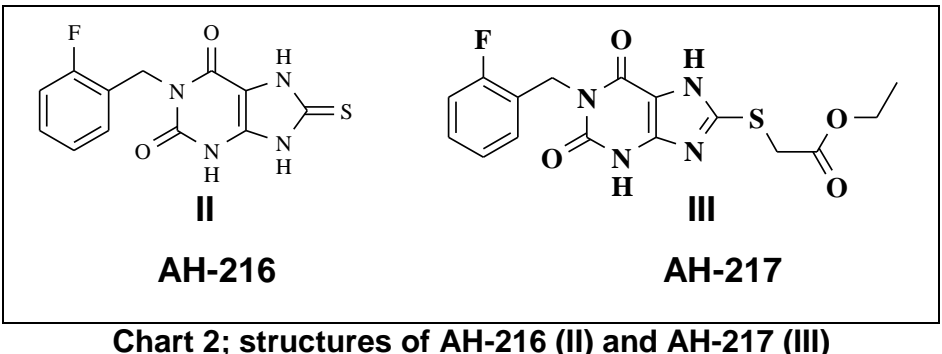

\section{MATERIALS AND METHODS}

\section{Chemistry}

\section{Materials}

Melting points were determined on an electrothermal melting point apparatus (Stuart Scientific, SMP3, Staffordshire, UK) and were uncorrected. Pre-coated silica gel plates (Kieselgel $0.25 \mathrm{~mm}, 60 \mathrm{G}$ F254, Merck, Darmstadt, Germany) were used for TLC monitoring of reactions using $\mathrm{CH}_{2} \mathrm{Cl}_{2} / \mathrm{CH}_{3} \mathrm{OH}$ 9:1 as a mobile phase. Visualization of the spots was effected using an ultraviolet lamp (Spectroline, model CM-10, Seattle, USA) $(\lambda=254 \mathrm{~nm})$ and/or iodine stain. IR spectra were carried out as $\mathrm{KBr}$ discs on a Shimadzu IR-470 Spectrophotometer (Shimadzu, Kyoto, Japan) at Faculty of Pharmacy, Assiut University. ${ }^{1} \mathrm{H}$ NMR spectra were performed on a JEOL JNMLA series FT NMR system (400 MHz, JEOL, Tokyo, Japan) at the Assiut University Unit of Trace Analyses or on a Varian spectrometer (300 MHz, Varian, CA, USA) at the unit of Microanalysis, Faculty of Science, Cairo University or on a Varian EM-360L NMR spectrometer $(60 \mathrm{MHz}, \mathrm{Varian}, \mathrm{CA}$, USA) at Faculty of Pharmacy, Assiut University, Assiut. Chemical shifts are expressed in $\delta$-values (ppm) relative to tetramethylsilane (TMS) as an internal standard using DMSO- $d_{6}$ as a solvent and deuterium oxide was used for the detection of exchangeable protons. Mass spectra were recorded with a Gas Chromatography Mass, Quadruple-2010 Plus (Shimadzu, Kyoto, Japan) at the unit of Microanalysis, Faculty of Science, Cairo University. ESI-HRMS were determined using Bruker Bio TOF III (ESI-TOF, Bruker, MA, USA) in the Genomic Research Centre, Academia Sinica, Taiwan. Elemental microanalyses were performed on a Vario elemental analyzer III (Vario, Hanau, Germany) at the unit of Microanalysis, Faculty of Science, Cairo University.

Compounds 6-amino-3-(2-substituted benzyl)-1H-pyrimidine-2,4-dione 1a,b, 2-[(1-substituted)-2,6-dioxo-2,3,6,7tetrahydro-1H-purin-8-ylsulfanyl]- $\mathrm{N}$-substituted-acetamide $\mathbf{6 a - g} \& \mathbf{7 a - g}$ and 1-substituted-8-[2-(4-substituted phenyl)-2oxoethylsulphanyl]-3,7-dihydro-1 $\mathrm{H}$-purine-2,6-diones 9a-g\&10a-g were prepared as reported in a recent pervious publication $^{29}$.

\section{Methods}

\section{General procedure for the preparation of 3-(2-Substituted benzyl)-6-(4-substituted phenyl)- 1H-thiazolo[2,3-f]purine -2,4-dione (11a-g, 12a-g)}

To a stirred freshly prepared polyphosphoric acid formed from phosphorus pentoxide $(8.00 \mathrm{~g})$ and phosphoric acid (6.00 $\mathrm{mL}$ ), the appropriate 1-substituted-8-[2-(4-substituted phenyl)-2-oxoethylsulfanyl]-3,7-dihydro-1 H-purine-2,6-diones (9a-g and $10 \mathrm{a}-\mathrm{g})(5.30 \mathrm{mmol})$ was added, and the reaction mixture was heated at $130-140^{\circ} \mathrm{C}$ for $5-6 \mathrm{~h}$ (except for 9 f and $10 \mathrm{f}$ were heated at $110-120^{\circ} \mathrm{C}$ for $7-8 \mathrm{~h}$ ). The reaction mixture was cooled, poured into ice-water and neutralized with saturated sodium carbonate solution. The precipitated solid was filtered, washed with water, dried, and crystallized from absolute ethanol to afford the target products 11a-g and 12a-g. 


\subsection{3-Benzyl-6-phenyl-1H-thiazolo[2,3-f]purine-2,4-dione 11a}

Buff crystals, m.p. 253-255드, yield 50\%, IR (KBr) ú (cm $\left.{ }^{-1}\right) 3320(\mathrm{~N}-\mathrm{H}) ; 3045$ (Ar-H); 2895 (C-H aliphatic); 1699,1650 $(\mathrm{C}=\mathrm{O}) ; 743,690$ (Ar-H); ${ }^{1} \mathrm{H}$ NMR $\left(400 \mathrm{MHz}, \mathrm{DMSO}-\mathrm{d}_{6}\right): \delta 4.89$ (s, 2H, N1-CH $), 7.14-7.54(\mathrm{~m}, 10 \mathrm{H}, \mathrm{Ar}-\mathrm{H}), 7.28(\mathrm{~s}, 1 \mathrm{H}, \mathrm{C7}-$ $\mathrm{H}), 12.25$ (s, $1 \mathrm{H}, \mathrm{N} 3-\mathrm{H}$, exchangeable with $\left.\mathrm{D}_{2} \mathrm{O}\right)$; Anal. Calc. (\%) for for $\mathrm{C}_{20} \mathrm{H}_{14} \mathrm{~N}_{4} \mathrm{O}_{2} \mathrm{~S}$ : C, 64.16; $\mathrm{H}, 3.77 ; \mathrm{N}, 14.96$; Found: C, 64.19; H, 4.00; N, 14.72 .

\subsection{3-Benzyl-6-(4-bromophenyl)-1H-thiazolo[2,3-f]purine-2,4-dione 11b}

Buff crystals, m.p. 298-2990 , yield 88\%, IR (KBr) ú $\left(\mathrm{cm}^{-1}\right) 3405(\mathrm{~N}-\mathrm{H}) ; 3090$ (Ar-H); 2990 (C-H aliphatic); 1696, 1664 $(\mathrm{C}=\mathrm{O})$; 806, 736, $710(\mathrm{Ar}-\mathrm{H}), 575(\mathrm{C}-\mathrm{Br}) ;{ }^{1} \mathrm{H}$ NMR $(400 \mathrm{MHz}$, DMSO-d $)$ : $\delta 4.91\left(\mathrm{~s}, 2 \mathrm{H}, \mathrm{N} 1-\mathrm{CH}_{2}\right), 7.14-7.26(\mathrm{~m}, 5 \mathrm{H}, \mathrm{Ar}-\mathrm{H})$, $7.33(\mathrm{~s}, 1 \mathrm{H}, \mathrm{C} 7-\mathrm{H}), 7.50$ (d, J = 8.4 Hz, 2H, 3',5'Ar-H), 7.60 (d, J = 8.4 Hz, 2H, 2',6'Ar-H), $12.27(\mathrm{~s}, 1 \mathrm{H}, \mathrm{N} 3-\mathrm{H}$, exchangeable with $\left.\mathrm{D}_{2} \mathrm{O}\right)$; ESI-HRMS $(\mathrm{m} / \mathrm{z}): 453.0028\left(\mathrm{M}^{+}+1\right)$ (calcd. 453.0015).

\subsection{3-Benzyl-6-(4-chlorophenyl)-1H-thiazolo[2,3-f]purine-2,4-dione 11c}

Buff crystals, m.p. 243-245ํㅡ, yield 68\%, IR (KBr) ú $\left(\mathrm{cm}^{-1}\right) 3400(\mathrm{~N}-\mathrm{H}) ; 3095(\mathrm{Ar}-\mathrm{H}) ; 2900$ (C-H aliphatic); 1695,1665 $(\mathrm{C}=\mathrm{O}) ; 1084$ (C-Cl); 830, 735, $694(\mathrm{Ar}-\mathrm{H}) ;{ }^{1} \mathrm{H}$ NMR (300 MHz, DMSO-d $)$ ): $\delta 5.12$ (s, 2H, N1-CH $), 7.11-7.32$ (m, 5H, Ar-H), $7.63\left(\mathrm{~d}, J=8.4 \mathrm{~Hz}, 2 \mathrm{H}, 3^{\prime}, 5^{\prime} \mathrm{Ar}-\mathrm{H}\right), 7.76(\mathrm{~s}, 1 \mathrm{H}, \mathrm{C} 7-\mathrm{H}), 8.21\left(\mathrm{~d}, J=8.4 \mathrm{~Hz}, 2 \mathrm{H}, 2^{\prime}, 6^{\prime} \mathrm{Ar}-\mathrm{H}\right), 12.00(\mathrm{~s}, 1 \mathrm{H}, \mathrm{N} 3-\mathrm{H}$, exchangeable with $\mathrm{D}_{2} \mathrm{O}$; El-MS: $\mathrm{m} / \mathrm{z}$ : $408.05\left(\mathrm{M}^{+}, 62.43 \%\right), 410.00\left(\mathrm{M}^{+}+2,25.32 \%\right), 91.05(100 \%)$. Anal. Calc. (\%) for $\mathrm{C}_{20} \mathrm{H}_{13} \mathrm{ClN}_{4} \mathrm{O}_{2} \mathrm{~S}$ : C, 58.75; H, 3.20; N, 13.70; Found: C, 58.67; H, 2.99; N, 13.60.

\subsection{3-Benzyl-6-(4-fluorophenyl)-1H-thiazolo[2,3-f]purine-2,4-dione 11d}

Buff crystals, m.p. 222-224ํㅡ, yield 63\%, IR (KBr) ú $\left(\mathrm{cm}^{-1}\right) 3400(\mathrm{~N}-\mathrm{H}) ; 3055(\mathrm{Ar}-\mathrm{H}) ; 2950$ (C-H aliphatic); 1700,1661 $(\mathrm{C}=\mathrm{O}) ; 1223(\mathrm{C}-\mathrm{F}) ; 832,732,694(\mathrm{Ar}-\mathrm{H}) ;{ }^{1} \mathrm{H}$ NMR $(60 \mathrm{MHz} \text {, DMSO-d })_{6}$ : $\delta 5.10\left(\mathrm{~s}, 2 \mathrm{H}, \mathrm{N} 1-\mathrm{CH}_{2}\right), 7.10(\mathrm{~s}, 1 \mathrm{H}, \mathrm{C} 7-\mathrm{H}), 7.20-$ $7.83(\mathrm{~m}, 9 \mathrm{H}, \mathrm{Ar}-\mathrm{H}), 12.50\left(\mathrm{~s}, 1 \mathrm{H}, \mathrm{N} 3-\mathrm{H}\right.$, exchangeable with $\left.\mathrm{D}_{2} \mathrm{O}\right)$; El-MS: m/z: $392.00\left(\mathrm{M}^{+}, 91.30 \%\right), 91.00$ (100\%); ESIHRMS $(\mathrm{m} / \mathrm{z}): 393.0813\left(\mathrm{M}^{+}+1\right)($ calcd. 393.0816).

\subsection{3-Benzyl-6-p-tolyl-1H-thiazolo[2,3-f]purine-2,4-dione 11e}

Buff crystals, m.p. 278-280ㅇ, yield 72\%, IR (KBr) ú $\left(\mathrm{cm}^{-1}\right) 3440(\mathrm{~N}-\mathrm{H}) ; 3090($ Ar-H); 2955 (C-H aliphatic); 1700,1651 $(\mathrm{C}=\mathrm{O}) ; 802,741,694(\mathrm{Ar}-\mathrm{H}) ;{ }^{1} \mathrm{H}$ NMR $\left(60 \mathrm{MHz}, \mathrm{DMSO}-d_{6}\right): \delta 2.43\left(\mathrm{~s}, 3 \mathrm{H}, \mathrm{CH}_{3}\right), 5.07\left(\mathrm{~s}, 2 \mathrm{H}, \mathrm{N} 1-\mathrm{CH}_{2}\right), 7.17-7.67(\mathrm{~m}, 10 \mathrm{H}$, Ar-H, C7-H), 12.40, (s, 1H, N3-H, exchangeable with $\mathrm{D}_{2} \mathrm{O}$ ); Anal. Calc. (\%) for $\mathrm{C}_{21} \mathrm{H}_{16} \mathrm{~N}_{4} \mathrm{O}_{2} \mathrm{~S}: \mathrm{C}, 64.93 ; \mathrm{H}, 4.15 ; \mathrm{N}, 14.42 ;$ Found: C, 64.81; H, 3.86; N, 14.28.

\subsection{3-Benzyl-6-(4-methoxyphenyl)-1H-thiazolo[2,3-f]purine-2,4-dione 11f}

Buff crystals, m.p. 200-202ㄷ, yield 58\%, IR (KBr) ú (cm $\left.{ }^{-1}\right) 3410(\mathrm{~N}-\mathrm{H}) ; 3055$ (Ar-H); 2900 (C-H aliphatic); 1697, 1651, 1620 (C=O); 1250, 1060 (C-O); 813, 743, 711 (Ar-H); ${ }^{1} \mathrm{H}$ NMR (60 MHz, DMSO-d 6 ): $\delta 3.87$ (s, 3H, OCH $\left.{ }_{3}\right), 5.07$ (s, $2 \mathrm{H}, \mathrm{N} 1-$ $\left.\mathrm{CH}_{2}\right), 6.98\left(\mathrm{~d}, J=8.2 \mathrm{~Hz}, 2 \mathrm{H}, 3^{\prime}, 5^{\prime} \mathrm{Ar}-\mathrm{H}\right), 7.10(\mathrm{~s}, 1 \mathrm{H}, \mathrm{C} 7-\mathrm{H}), 7.30(\mathrm{~s}, 5 \mathrm{H}, \mathrm{Ar}-\mathrm{H}), 7.57$ (d, J = 8.2 Hz, 2H, 2',6'Ar-H), 12.33 $\left(\mathrm{s}, 1 \mathrm{H}, \mathrm{N} 3-\mathrm{H}\right.$, exchangeable with $\left.\mathrm{D}_{2} \mathrm{O}\right)$; ESI-HRMS $(\mathrm{m} / \mathrm{z}): 405.1012\left(\mathrm{M}^{+}+1\right)$ (calcd. 405.1016).

\subsection{3-Benzyl-6-(4-nitrophenyl)-1H-thiazolo[2,3-f]purine-2,4-dione 11g}

Buff crystals, m.p. 299-301ํㅡ, yield 85\%, IR (KBr) ú (cm $\left.{ }^{-1}\right) 3445(\mathrm{~N}-\mathrm{H}) ; 3115(\mathrm{Ar}-\mathrm{H}) ; 2950$ (C-H aliphatic); 1698,1653 $(\mathrm{C}=\mathrm{O}) ; 1503,1342\left(\mathrm{NO}_{2}\right) ; 833,767,702(\mathrm{Ar}-\mathrm{H}) ;{ }^{1} \mathrm{H}$ NMR $\left(60 \mathrm{MHz}, \mathrm{DMSO}-d_{6}\right)$ : $\delta 5.03\left(\mathrm{~s}, 2 \mathrm{H}, \mathrm{N} 1-\mathrm{CH}_{2}\right), 7.23(\mathrm{~s}, 5 \mathrm{H}, \mathrm{Ar}-\mathrm{H})$, $7.33(\mathrm{~s}, 1 \mathrm{H}, \mathrm{C} 7-\mathrm{H}), 7.88\left(\mathrm{~d}, J=8.8 \mathrm{~Hz}, 2 \mathrm{H}, 2^{\prime}, 6^{\prime} \mathrm{Ar}-\mathrm{H}\right), 8.32\left(\mathrm{~d}, J=8.8 \mathrm{~Hz}, 2 \mathrm{H}, 3^{\prime}, 5^{\prime} \mathrm{Ar}-\mathrm{H}\right), 12.50(\mathrm{~s}, 1 \mathrm{H}, \mathrm{N} 3-\mathrm{H}$, exchangeable with $\mathrm{D}_{2} \mathrm{O}$ ); ESI-HRMS $(\mathrm{m} / \mathrm{z})$ : $420.0762\left(\mathrm{M}^{+}+1\right)$ (calcd. 420.0761); Anal. Calc. (\%) for $\mathrm{C}_{20} \mathrm{H}_{13} \mathrm{~N}_{5} \mathrm{O}_{4} \mathrm{~S}$ : $\mathrm{C}$ 57.27; H, 3.12; N, 16.70; Found: C, 57.02; H, 3.38; N, 16.60.

\subsection{3-(2-Fluorobenzyl)-6-phenyl-1H-thiazolo[2,3-f]purine-2,4-dione 12a}

Buff crystals, m.p. 326-328으, yield 95\%, IR (KBr) ú (cm $\left.{ }^{-1}\right) 3420(\mathrm{~N}-\mathrm{H}) ; 3035(\mathrm{Ar}-\mathrm{H}) ; 2865$ (C-H aliphatic); 1702,1644 $(\mathrm{C}=\mathrm{O}) ; 1219$ (C-F); 739, 691 (Ar-H); ${ }^{1} \mathrm{H}$ NMR (60 MHz, DMSO-d $)$ : $\delta 4.93\left(\mathrm{~s}, 2 \mathrm{H}, \mathrm{N} 1-\mathrm{CH}_{2}\right), 6.53-7.57(\mathrm{~m}, 9 \mathrm{H}, \mathrm{Ar}-\mathrm{H}), 7.40$ (s, $1 \mathrm{H}, \mathrm{C} 7-\mathrm{H}), 12.50\left(\mathrm{~s}, 1 \mathrm{H}, \mathrm{N} 3-\mathrm{H}\right.$, exchangeable with $\left.\mathrm{D}_{2} \mathrm{O}\right)$; Anal. Calc. (\%) for $\mathrm{C}_{20} \mathrm{H}_{13} \mathrm{FN}_{4} \mathrm{O}_{2} \mathrm{~S}: \mathrm{C}, 61.22 ; \mathrm{H}, 3.34 ; \mathrm{N}$, 14.28; Found: C, $61.00 ; \mathrm{H}, 3.58 ; \mathrm{N}, 14.32$.

\subsection{6-(4-Bromophenyl)-3-(2-fluorobenzyl)-1H-thiazolo[2,3-f]purine-2,4-dione} $12 b$

Buff crystals, m.p. 313-315드, yield 67\%, IR (KBr) ú (cm $\left.{ }^{-1}\right) 3380(\mathrm{~N}-\mathrm{H}) ; 3110(\mathrm{Ar}-\mathrm{H}) ; 2940$ (C-H aliphatic); 1696,1656 $(\mathrm{C}=\mathrm{O}) ; 1557(\mathrm{~N}-\mathrm{H}) ; 1220(\mathrm{C}-\mathrm{F}) ; 809,748(\mathrm{Ar}-\mathrm{H}) ; 540(\mathrm{C}-\mathrm{Br}) ;{ }^{1} \mathrm{H}$ NMR $\left(60 \mathrm{MHz}, \mathrm{DMSO}-d_{6}\right): \delta 5.07\left(\mathrm{~s}, 2 \mathrm{H}, \mathrm{N} 1-\mathrm{CH}_{2}\right), 6.77-$ $7.77(\mathrm{~m}, 8 \mathrm{H}, \mathrm{Ar}-\mathrm{H}), 7.30(\mathrm{~s}, 1 \mathrm{H}, \mathrm{C} 7-\mathrm{H}), 12.20$ (br s, $1 \mathrm{H}, \mathrm{N} 3-\mathrm{H}$, exchangeable with $\left.\mathrm{D}_{2} \mathrm{O}\right)$; El-MS: $m / z: 469.90\left(\mathrm{M}^{+}, 87.15 \%\right)$, $471.90\left(\mathrm{M}^{+}+2,77.97 \%\right), 109.00$ (100\%); Anal. Calc. (\%) for $\mathrm{C}_{20} \mathrm{H}_{12} \mathrm{BrFN}_{4} \mathrm{O}_{2} \mathrm{~S}: \mathrm{C}, 50.97 ; \mathrm{H}, 2.57 ; \mathrm{N}, 11.89 ; \mathrm{S}, 6.80 ;$ Found: C, 51.18; H, 2.60; N, 11.74; S, 6.57 .

\subsection{6-(4-Chlorophenyl)-3-(2-fluorobenzyl)-1H-thiazolo[2,3-f]purine-2,4-dione} $12 \mathrm{c}$ 
Buff crystals, m.p. 195-197º $\mathrm{C}$, yield 50\%, IR (KBr) ú $\left(\mathrm{cm}^{-1}\right) 3470(\mathrm{~N}-\mathrm{H}) ; 3045(\mathrm{Ar}-\mathrm{H}) ; 2895$ (C-H aliphatic); 1694,1656 $(\mathrm{C}=\mathrm{O}) ; 1219(\mathrm{C}-\mathrm{F}) ; 1083(\mathrm{C}-\mathrm{Cl}) ; 830,747(\mathrm{Ar}-\mathrm{H}) ;{ }^{1} \mathrm{H}$ NMR $\left(60 \mathrm{MHz}, \mathrm{DMSO}-d_{6}\right)$ : $\delta 5.10\left(\mathrm{~s}, 2 \mathrm{H}, \mathrm{N} 1-\mathrm{CH}_{2}\right), 7.00-7.80(\mathrm{~m}, 9 \mathrm{H}$, $\mathrm{Ar}-\mathrm{H}, \mathrm{C} 7-\mathrm{H}$ ), 12.30 (br s, $1 \mathrm{H}, \mathrm{N} 3-\mathrm{H}$, exchangeable with $\mathrm{D}_{2} \mathrm{O}$ ); Anal. Calc. (\%) for $\mathrm{C}_{20} \mathrm{H}_{12} \mathrm{ClFN}_{4} \mathrm{O}_{2} \mathrm{~S}$ : C, 56.28; $\mathrm{H}, 2.83 ; \mathrm{N}$, 13.13; Found: C, 56.00; H, 2.73; N, 12.88.

\subsection{3-(2-Fluorobenzyl)-6-(4-fluorophenyl)-1H-thiazolo[2,3-f]purine-2,4-dione $12 \mathrm{~d}$}

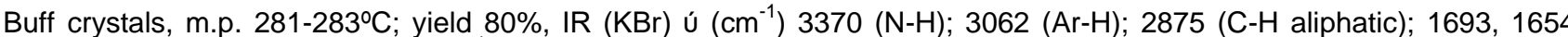
$(\mathrm{C}=\mathrm{O}) ; 1222(\mathrm{C}-\mathrm{F}) ; 819,746(\mathrm{Ar}-\mathrm{H}) ;{ }^{1} \mathrm{H}$ NMR $\left(60 \mathrm{MHz}, \mathrm{DMSO}-d_{6}\right): \delta 5.07$ (s, 2H, N1-CH $), 7.00-7.93(\mathrm{~m}, 9 \mathrm{H}, \mathrm{Ar}-\mathrm{H}, \mathrm{C} 7-\mathrm{H})$, 12.30 (s, $1 \mathrm{H}$, N3-H, exchangeable with $\mathrm{D}_{2} \mathrm{O}$ ); Anal. Calc. (\%) for $\mathrm{C}_{20} \mathrm{H}_{12} \mathrm{~F}_{2} \mathrm{~N}_{4} \mathrm{O}_{2} \mathrm{~S}$ : C, 58.53; $\mathrm{H}, 2.95 ; \mathrm{N}, 13.65 ; \mathrm{S}, 7.81 ;$ Found: C, 58.26; H, 3.18; N, 13.42; S, 7.34.

\subsection{3-(2-Fluorobenzyl)-6-p-tolyl-1H-thiazolo[2,3-f]purine-2,4-dione 12e}

Buff crystals, m.p. 295-297으; yield 89\%, IR (KBr) ú $\left(\mathrm{cm}^{-1}\right) 3360(\mathrm{~N}-\mathrm{H}) ; 3045$ (Ar-H); 2945 (C-H aliphatic); 1697, 1654 $(\mathrm{C}=\mathrm{O}) ; 1221(\mathrm{C}-\mathrm{F}) ; 809,745(\mathrm{Ar}-\mathrm{H}) ;{ }^{1} \mathrm{H}$ NMR (300 MHz, DMSO-d 6 ): $\delta 2.35$ (s, 3H, CH $\left.\mathrm{CH}_{3}\right), 4.96\left(\mathrm{~s}, 2 \mathrm{H}, \mathrm{N} 1-\mathrm{CH}_{2}\right), 6.97-7.19$ $(\mathrm{m}, 5 \mathrm{H}, \mathrm{Ar}-\mathrm{H}), 7.22\left(\mathrm{~d}, J=8.2 \mathrm{~Hz}, 2 \mathrm{H}, 3^{\prime}, 5^{\prime} \mathrm{Ar}-\mathrm{H}\right), 7.24(\mathrm{~s}, 1 \mathrm{H}, \mathrm{C} 7-\mathrm{H}), 7.42\left(\mathrm{~d}, J=8.2 \mathrm{~Hz}, 2 \mathrm{H}, 2^{\prime}, 6{ }^{\prime} \mathrm{Ar}-\mathrm{H}\right), 12.27$ (br s, $1 \mathrm{H}$, $\mathrm{N} 3-\mathrm{H}$, exchangeable with $\mathrm{D}_{2} \mathrm{O}$ ); El-MS: $\mathrm{m} / \mathrm{z}$ : $406.00\left(\mathrm{M}^{+}, 100 \%\right.$; Anal. Calc. (\%) for $\mathrm{C}_{21} \mathrm{H}_{15} \mathrm{FN}_{4} \mathrm{O}_{2} \mathrm{~S}$ : $\mathrm{C}, 62.06 ; \mathrm{H}, 3.72 ; \mathrm{N}$, 13.79; S, 7.89; Found: C, 61.94; H, 3.54; N, 13.46; S, 7.69.

\subsection{3-(2-Fluorobenzyl)-6-(4-methoxyphenyl)-1H-thiazolo[2,3-f]purine-2,4-dione $12 f$}

Buff crystals, m.p. 242-244으; yield 51\%, IR (KBr) ú $\left(\mathrm{cm}^{-1}\right) 3435(\mathrm{~N}-\mathrm{H}) ; 3045$ (Ar-H); 2930 (C-H aliphatic); 1697, 1663 $(\mathrm{C}=\mathrm{O})$; 1249, 1025 (C-O); 1212 (C-F); 818, 755 (Ar-H); ${ }^{1} \mathrm{H}$ NMR (60 MHz, DMSO-d6): $\delta 3.83$ (s, 3H, OCH $), 5.07$ (s, $2 \mathrm{H}$, $\left.\mathrm{N} 1-\mathrm{CH}_{2}\right), 6.67-7.27(\mathrm{~m}, 7 \mathrm{H}, \mathrm{Ar}-\mathrm{H}, \mathrm{C} 7-\mathrm{H}), 7.47\left(\mathrm{~d}, \mathrm{~J}=8.6 \mathrm{~Hz}, 2 \mathrm{H}, 2^{\prime}, 6^{\prime} \mathrm{Ar}-\mathrm{H}\right), 12.33$ (br s, 1H, N3-H, exchangeable with $\left.\mathrm{D}_{2} \mathrm{O}\right)$; ESI-HRMS $(\mathrm{m} / \mathrm{z}): 423.0925\left(\mathrm{M}^{+}+1\right)$ (calcd. 423.0922)

\subsection{3-(2-Fluorobenzyl)-6-(4-nitrophenyl)-1H-thiazolo[2,3-f]purine-2,4-dione 12g}

Buff crystals, m.p. 300-302을 yield 59\%, IR (KBr) ú $\left(\mathrm{cm}^{-1}\right) 3390(\mathrm{~N}-\mathrm{H}) ; 3100(\mathrm{Ar}-\mathrm{H}) ; 2950$ (C-H aliphatic); 1701,1662 $(\mathrm{C}=\mathrm{O}) ; 1537,1333\left(\mathrm{NO}_{2}\right) ; 1211(\mathrm{C}-\mathrm{F}) ; 820,746(\mathrm{Ar}-\mathrm{H}) ;{ }^{1} \mathrm{H}$ NMR $\left(400 \mathrm{MHz}, \mathrm{DMSO}-\mathrm{d}_{6}\right): \delta 4.93\left(\mathrm{~s}, 2 \mathrm{H}, \mathrm{N} 1-\mathrm{CH}_{2}\right), 6.63-7.23$ $(\mathrm{m}, 4 \mathrm{H}, \mathrm{Ar}-\mathrm{H}), 7.27(\mathrm{~s}, 1 \mathrm{H}, \mathrm{C} 7-\mathrm{H}), 7.76\left(\mathrm{~d}, \mathrm{~J}=8.4 \mathrm{~Hz}, 2 \mathrm{H}, 2^{\prime}, 6^{\prime} \mathrm{Ar}-\mathrm{H}\right), 8.20\left(\mathrm{~d}, \mathrm{~J}=8.4 \mathrm{~Hz}, 2 \mathrm{H}, 3^{\prime}, 5^{\prime} \mathrm{Ar}-\mathrm{H}\right), 12.30(\mathrm{br} \mathrm{s}, 1 \mathrm{H}$, $\mathrm{N} 3-\mathrm{H}$, exchangeable with $\left.\mathrm{D}_{2} \mathrm{O}\right)$; ESI-HRMS $(\mathrm{m} / \mathrm{z}): 438.0666\left(\mathrm{M}^{+}+1\right)($ calcd. 438.0667$)$

\subsubsection{3-substituted benzyl-5-methyl-7-substituted-1H-pyrido[2,3-d]pyrimidine-2,4- dione 13a-c,14 a-c}

6-Amino-3-(2-substituted benzyl)-1 H-pyrimidine-2,4-dione $\mathbf{1 a}$ or $\mathbf{1 b}(1.50 \mathrm{mmol})$ and the appropriate acylacetone derivative $(1.50 \mathrm{mmol})$ were dissolved in glacial acetic acid $(10.00 \mathrm{~mL})$ and the solution was refluxed for $3-4 \mathrm{~h}$. The reaction mixture was poured in an ice-cooled water and was made basic to litmus with ammonia solution (25\%). The precipitate was filtered, washed with water and recrystallized from absolute ethanol to afford the target products 13a-c and 14a-c.

\subsection{3-Benzyl-5,7-dimethyl-1H-pyrido[2,3-d]pyrimidine-2,4-dione 13a}

Yellow crystals, m.p. 248-250두 yield 52\%, IR (KBr) ú (cm $\left.{ }^{-1}\right) 3395(\mathrm{~N}-\mathrm{H}) ; 3185(\mathrm{Ar}-\mathrm{H}) ; 2960$ (C-H aliphatic); 1700,1656 $(\mathrm{C}=\mathrm{O}) ; 750,700(\mathrm{Ar}-\mathrm{H}) ;{ }^{1} \mathrm{H}$ NMR $\left(60 \mathrm{MHz}, \mathrm{DMSO}-d_{6}\right): \delta 2.57\left(\mathrm{~s}, 3 \mathrm{H}, \mathrm{CH}_{3}\right), 2.72\left(\mathrm{~s}, 3 \mathrm{H}, \mathrm{CH}_{3}\right), 5.10\left(\mathrm{~s}, 2 \mathrm{H}, \mathrm{N} 3-\mathrm{CH}_{2}\right), 6.63$ (s, $1 \mathrm{H}, \mathrm{C} 6-\mathrm{H}), 7.00-7.57(\mathrm{~m}, 5 \mathrm{H}, \mathrm{Ar}-\mathrm{H}), 10.37\left(\mathrm{~s}, 1 \mathrm{H}, \mathrm{N} 1-\mathrm{H}\right.$, exchangeable with $\left.\mathrm{D}_{2} \mathrm{O}\right) ; \mathrm{El}-\mathrm{MS}: \mathrm{m} / \mathrm{z}: 280.90\left(\mathrm{M}^{+}, 100 \%\right) ;$ Anal. Calc. (\%) for $\mathrm{C}_{16} \mathrm{H}_{15} \mathrm{~N}_{3} \mathrm{O}_{2}$ : C, 68.31; $\mathrm{H}, 5.37 ; \mathrm{N}, 14.94$; Found: $\mathrm{C}, 68.22 ; \mathrm{H}, 5.71 ; \mathrm{N}, 14.77$.

\subsection{3-Benzyl-5-methyl-7-phenyl-1H-pyrido[2,3-d]pyrimidine-2,4-dione 13b}

Yellow crystals, m.p. 187-189C; yield 55\%, IR (KBr) ú $\left(\mathrm{cm}^{-1}\right) 3390(\mathrm{~N}-\mathrm{H}) ; 3050(\mathrm{Ar}-\mathrm{H}) ; 2950(\mathrm{C}-\mathrm{H}$ aliphatic); 1690,1647 $(\mathrm{C}=\mathrm{O}) ; 750,691(\mathrm{Ar}-\mathrm{H}) ;{ }^{1} \mathrm{H}$ NMR $\left(60 \mathrm{MHz}, \mathrm{DMSO}-d_{6}\right): \delta 2.73\left(\mathrm{~s}, 3 \mathrm{H}, \mathrm{CH}_{3}\right), 5.10\left(\mathrm{~s}, 2 \mathrm{H}, \mathrm{N} 3-\mathrm{CH}_{2}\right), 6.67(\mathrm{~s}, 1 \mathrm{H}, \mathrm{C} 6-\mathrm{H}), 6.93-$ $8.90(\mathrm{~m}, 10 \mathrm{H}, \mathrm{Ar}-\mathrm{H}), 10.83\left(\mathrm{~s}, 1 \mathrm{H}, \mathrm{N1}-\mathrm{H}\right.$, exchangeable with $\mathrm{D}_{2} \mathrm{O}$ ); Anal. Calc. (\%) for $\mathrm{C}_{21} \mathrm{H}_{17} \mathrm{~N}_{3} \mathrm{O}_{2}: \mathrm{C}, 73.45 ; \mathrm{H}, 4.99 ; \mathrm{N}$, 12.24; Found: C, $73.11 ; \mathrm{H}, 4.56 ; \mathrm{N}, 12.30$.

\subsubsection{3. \\ 3-Benzyl-7-(4-chlorophenyl)-5-methyl-1H-pyrido[2,3-d]pyrimidine-2,4- dione 13c}

Yellow crystals, m.p. 167-1690 ; yield 55\%, IR (KBr) ú (cm $\left.{ }^{-1}\right) 3375$ (N-H); 3100 (Ar-H); 2950 (C-H aliphatic); 1697,1645 $(\mathrm{C}=\mathrm{O}) ; 1084(\mathrm{C}-\mathrm{Cl}) ; 811,760,691(\mathrm{Ar}-\mathrm{H}) ;{ }^{1} \mathrm{H}$ NMR $\left(400 \mathrm{MHz}, \mathrm{DMSO}-d_{6}\right): \delta 2.75\left(\mathrm{~s}, 3 \mathrm{H}, \mathrm{CH}_{3}\right), 5.05(\mathrm{~s}, 2 \mathrm{H}, \mathrm{N} 3-\mathrm{CH}), 7.24$ $7.33(\mathrm{~m}, 5 \mathrm{H}, \mathrm{Ar}-\mathrm{H}), 7.61$ (d, J = 8.4 Hz, 2H, 3',5'Ar-H), $7.75(\mathrm{~s}, 1 \mathrm{H}, \mathrm{C} 6-\mathrm{H}), 8.19\left(\mathrm{~d}, \mathrm{~J}=8.4 \mathrm{~Hz}, 2 \mathrm{H}, 2^{\prime}, 6^{\prime} \mathrm{Ar}-\mathrm{H}\right), 11.99(\mathrm{~s}, 1 \mathrm{H}$ $\mathrm{N} 1-\mathrm{H}$, exchangeable with $\left.\mathrm{D}_{2} \mathrm{O}\right)$; ESI-HRMS $(\mathrm{m} / \mathrm{z}): 378.1014\left(\mathrm{M}^{+}+1\right)$ (calcd. 378.1004$)$.

\subsection{3-(2-Fluorobenzyl)-5,7-dimethyl-1H-pyrido[2,3-d]pyrimidine-2,4-dione 14a}


Yellow crystals, m.p. 253-255웅 yield 61\%, IR (KBr) ú (cm $\left.{ }^{-1}\right) 3350(\mathrm{~N}-\mathrm{H}) ; 3080(\mathrm{Ar}-\mathrm{H}) ; 2995(\mathrm{C}-\mathrm{H}$ aliphatic); 1709,1651 $(\mathrm{C}=\mathrm{O}) ; 1222(\mathrm{C}-\mathrm{F}) ; 750(\mathrm{Ar}-\mathrm{H}) ;{ }^{1} \mathrm{H}$ NMR $\left(300 \mathrm{MHz}, \mathrm{DMSO}-d_{6}\right): \delta 2.51\left(\mathrm{~s}, 3 \mathrm{H}, \mathrm{CH}_{3}\right), 2.63\left(\mathrm{~s}, 3 \mathrm{H}, \mathrm{CH}_{3}\right), 5.08(\mathrm{~s}, 2 \mathrm{H}, \mathrm{N} 3-$ $\left.\mathrm{CH}_{2}\right), 6.89(\mathrm{~s}, 1 \mathrm{H}, \mathrm{C} 6-\mathrm{H}), 7.10-7.30(\mathrm{~m}, 4 \mathrm{H}, \mathrm{Ar}-\mathrm{H}), 11.85\left(\mathrm{~s}, 1 \mathrm{H}, \mathrm{N} 1-\mathrm{H}\right.$, exchangeable with $\left.\mathrm{D}_{2} \mathrm{O}\right) ; \mathrm{El}-\mathrm{MS}: \mathrm{m} / \mathrm{z}: 299.00\left(\mathrm{M}^{+}\right.$, 48.23\%), 109.00 (100\%); Anal. Calc. (\%) for $\mathrm{C}_{16} \mathrm{H}_{14} \mathrm{FN}_{3} \mathrm{O}_{2}$ : C, 64.21; H, 4.71; N, 14.04; Found: C, 64.00; $\mathrm{H}, 4.46 ; \mathrm{N}, 14.35$.

\subsection{3-(2-Fluorobenzyl)-5-methyl-7-phenyl-1H-pyrido[2,3-d]pyrimidine-2,4-dione $14 \mathrm{~b}$}

Yellow crystals, m.p. 149-151ํㅡ; yield 58\%, IR (KBr) ú (cm ${ }^{-1} 3435(\mathrm{~N}-\mathrm{H}) ; 3085$ (Ar-H); 2950 (C-H aliphatic); 1710,1652 $(\mathrm{C}=\mathrm{O}) ; 1228(\mathrm{C}-\mathrm{F}) ; 766,690(\mathrm{Ar}-\mathrm{H}) ;{ }^{1} \mathrm{H}$ NMR $\left(60 \mathrm{MHz}, \mathrm{DMSO}-d_{6}\right): \delta 2.03\left(\mathrm{~s}, 3 \mathrm{H}, \mathrm{CH}_{3}\right), 5.73\left(\mathrm{~s}, 2 \mathrm{H}, \mathrm{N} 3-\mathrm{CH}_{2}\right), 7.33-8.03$ $(\mathrm{m}, 10 \mathrm{H}, \mathrm{Ar}-\mathrm{H}, \mathrm{C} 6-\mathrm{H}), 10.33$ (s, $1 \mathrm{H}, \mathrm{N} 1-\mathrm{H}$, exchangeable with $\left.\mathrm{D}_{2} \mathrm{O}\right)$; El-MS: $\mathrm{m} / \mathrm{z}$ : $361.00\left(\mathrm{M}^{+}, 9.86 \%\right), 84.00(100 \%)$; Anal. Calc. (\%) for $\mathrm{C}_{21} \mathrm{H}_{16} \mathrm{FN}_{3} \mathrm{O}_{2}$ : C, 69.80; $\mathrm{H}, 4.46 ; \mathrm{N}, 11.63$; Found: $\mathrm{C}, 69.60 ; \mathrm{H}, 4.59 ; \mathrm{N}, 11.28$.

\subsection{7-(4-Chlorophenyl)-3-(2-fluorobenzyl)-5-methyl-1H-pyrido[2,3- d]pyrimidine-2,4-dione 14c}

Yellow crystals, m.p. 116-118우 yield 50\%, IR (KBr) ú $\left(\mathrm{cm}^{-1}\right) 3430(\mathrm{~N}-\mathrm{H}) ; 3050(\mathrm{Ar}-\mathrm{H}) ; 2995$ (C-H aliphatic); 1702,1650 $(\mathrm{C}=\mathrm{O})$; 1221 (C-F); 1083 (C-Cl); 839, 754 (Ar-H); ${ }^{1} \mathrm{H}$ NMR (300 MHz, DMSO-d6): $\delta 2.76$ (s, 3H, CH $)_{3}, 5.12$ (s, 2H, N3$\left.\mathrm{CH}_{2}\right), 7.09-7.32(\mathrm{~m}, 5 \mathrm{H}, \mathrm{Ar}-\mathrm{H}), 7.63\left(\mathrm{~d}, \mathrm{~J}=8.4 \mathrm{~Hz}, 2 \mathrm{H}, 3^{\prime}, 5^{\prime} \mathrm{Ar}-\mathrm{H}\right), 7.76(\mathrm{~s}, 1 \mathrm{H}, \mathrm{C} 7-\mathrm{H}), 8.20\left(\mathrm{~d}, J=8.4 \mathrm{~Hz}, 2 \mathrm{H}, 2^{\prime}, 6^{\prime} \mathrm{Ar}-\mathrm{H}\right)$, $11.98\left(\mathrm{~s}, 1 \mathrm{H}, \mathrm{N} 1-\mathrm{H}\right.$, exchangeable with $\left.\mathrm{D}_{2} \mathrm{O}\right)$; El-MS: $\mathrm{m} / \mathrm{z}$ : $394.90\left(\mathrm{M}^{+}, 26.90 \%\right), 396.90\left(\mathrm{M}^{+}+2,10.13 \%\right), 109.00(100 \%) ;$ Anal. Calc. (\%) for $\mathrm{C}_{21} \mathrm{H}_{15} \mathrm{ClFN}_{3} \mathrm{O}_{2}$ : C, 63.72; $\mathrm{H}, 3.82 ; \mathrm{N}, 10.62$; Found: $\mathrm{C}, 63.83 ; \mathrm{H}, 4.00 ; \mathrm{N}, 10.28$.

\subsection{Anticancer Activity}

The anticancer activity was carried out at the National Cancer Institute, Cairo University. 4a, 4b, 6c, 7c, 9c, 10a, 10c, 12a, 13a, 13b and 14a, were chosen to test for their anticancer activity against human breast carcinoma MCF-7 cell line in comparison to Doxorubicin as a reference drug.

\subsubsection{Drugs, chemicals and reagents}

The referent anticancer drug Doxorubicin was purchased from Sigma (Perth, Western Australia) and used as positive control. Test compounds were dissolved in $20 \%$ DMSO in $1 \mathrm{mg} / \mathrm{mL}$ concentration. Serial dilution of the compounds were made to reach final concentrations of $5,12.50,25,50 \mu \mathrm{g} / \mathrm{mL}$. All the chemical used are of high analytical grade and were obtained from Sigma-Aldrich.

\subsubsection{Tumor cell lines and culture conditions}

The breast carcinoma cell line was obtained frozen in liquid nitrogen $\left(-180^{\circ} \mathrm{C}\right)$ from the American Type Culture Collection and was maintained in the National Cancer Institute, Cairo, Egypt, by serial sub-culturing. MCF-7 was grown as a monolayer culture in RPMI-1640 medium supplemented in 10\% Fetal Bovine Serum (FBS) and $1 \%$ Penicillin/Streptomycin. The cell line was then incubated at $37^{\circ} \mathrm{C}, 5 \% \mathrm{CO}_{2}, 95 \%$ air and high humidity atmosphere in water jacketed incubator (Revco, GS laboratory equipment, RCO 3000 TVBB, USA). Cell line was regularly subcultures to maintain in the exponential growth phase. Sterile conditions were strictly attained by working under equipped laminar flow (Microflo Laminar flow cabinet, Hamsphire SPP 105a, USA).

\subsubsection{Cytotoxic activity of test compounds}

The cytotoxicity of the tested compounds were tested on MCF-7 cell line using SRB assay according to a reported procedure ${ }^{30}$. Cells were seeded in 96 -well microtiter plates and left to attach to the plates for $24 \mathrm{~h}$. After $24 \mathrm{~h}$, cells were incubated with the appropriate concentration ranges of drugs $(0.00,5.00,12.50,25.00$ and $50.00 \mu \mathrm{g} / \mathrm{mL})$ and incubation was continued for 24,48 and $72 \mathrm{~h}$. Control cells were treated with vehicle alone. For each drug concentration, 4 wells were used. Following 24, 48 and $72 \mathrm{~h}$ treatment, the cells were fixed with $50 \mu \mathrm{L}$ cold $50 \%$ trichloroacetic acid for $1 \mathrm{~h}$ at $4^{\circ} \mathrm{C}$. Wells were washed 5 times with distilled water and stained for $30 \mathrm{~min}$ at room temperature with $50 \mu \mathrm{L} 0.4 \%$ Sulphorhodamine-B (SRB) dissolved in 1\% acetic acid, then washed 4 times with $1 \%$ acetic acid. The plates were airdried and the dye was solubilized with $100 \mu \mathrm{L} /$ well of $10 \mathrm{mM}$ tris base $(\mathrm{pH} 10.5)$ for $5 \mathrm{~min}$ on a shaker (Orbital shaker OS 20, Boeco, Germany) at $1600 \mathrm{rpm}$. The optical density (O.D.) of each well was measured spectrophotometrically at 564 $\mathrm{nm}$ with an enzyme linked immunosorbent assay (ELIZA) microplate reader (Meter tech. $\Sigma$ 960, U.S.A.). The mean background absorbance was automatically subtracted and mean values of each drug concentration was calculated. The percentage of cell survival was calculated as follows: Survival fraction = O.D. (treated cells)/O.D. (control cells). The IC 50 values (the concentrations of thymoquinone required to produce $50 \%$ inhibition of cell growth) were calculated using commercially available software (GraphPad Prizm). All experiments were carried out in triplicate and the data are listed in 
Table 1: Cytotoxic activity of compounds $4 a, 4 b, 6 c, 7 c, 9 c, 10 a, 10 c, 12 a, 13 a, 13 b, 14 a$ and Doxorubicin on MCF-7 cell line.

\begin{tabular}{|c|c|c|c|}
\hline $\begin{array}{c}\text { Compd. } \\
\text { No. }\end{array}$ & $\begin{array}{c}\mathrm{IC}_{50} \\
\mu \mathrm{mol} / \mathrm{mL}\end{array}$ & $\begin{array}{c}\text { Compd. } \\
\text { No. }\end{array}$ & $\begin{array}{c}\mathrm{IC}_{50} \\
\mu \mathrm{mol} / \mathrm{mL}\end{array}$ \\
\hline \hline Doxorubicin & 7.73 & $\mathbf{1 0 a}$ & 56.82 \\
\hline $\mathbf{4 a}$ & 15.64 & $\mathbf{1 0 c}$ & 10.04 \\
\hline $\mathbf{4 b}$ & 19.95 & $\mathbf{1 2 a}$ & 14.00 \\
\hline 6c & 18.35 & $\mathbf{1 3 a}$ & 13.41 \\
\hline $\mathbf{7 c}$ & 8.87 & $\mathbf{1 3 b}$ & 11.98 \\
\hline 9c & 7.25 & $\mathbf{1 4 a}$ & 69.54 \\
\hline
\end{tabular}

\subsection{Molecular Modeling}

Pharmacophore studies were carried out on Dell Precision ${ }^{\mathrm{TM}}$ T3600 Workstation [Intel Xeon E5-1660 3.3GHz, 16GB 1600MHz DDR3, ECC RDIMM 1TB (7200RPM), 1GB NVIDIA Quadro 2000, Windows 7 Professional (64 Bit)]. The training set was built from 30 compounds using the Molecular Operating Environment (MOE) package version 2011.10 (Chemical Computing Group, Inc. Molecular Operating Environment (MOE). CCG, Montreal, Canada. 2011). A commonfeatures pharmacophore model was derived with the HipHop module of Catalyst using the Accelrys Discovery Studio 2.5 (DS) software. For all compounds in the training set, energy minimization process was performed with CHARMM forcefield. Poling algorithm ${ }^{31}$ was applied to generate a maximum of 255 diverse conformations with the energy threshold of $20 \mathrm{kcal} \mathrm{mol}^{-1}$ above the calculated energy minimum for every compound in the dataset. These conformers were generated using Diverse Conformer Generation protocol running with Best/Flexible conformer generation option as available in DS. The most active compound (M1) was considered as 'reference compound' specifying a 'Principal' value of 2 and a 'MaxOmitFeat' value of 0 , meaning its structure and conformation would have the strongest influence in the model building phase. The 'Principal' value and 'MaxOmitFeat' value for the remaining active compounds were set to 1 and 1 , respectively. The inactive compounds were given a 'Principal' value and 'MaxOmitFeat' value of 0 and 1, respectively.

Feature Mapping protocol was used to identify the common chemical groups present in the training set compounds. As predicted, hydrogen bond acceptor (HBA), hydrogen bond donor (HBD), hydrophobic (HY), and RING_AROMATIC (RA), Positive_Ionizable, Negative_lonizable features were selected during the pharmacophore generation. Misses specifies the number of molecules that do not have to map to all features in the hypothesis. FeatureMisses, the number of maximal molecules which do not have to map to each feature in generated hypothesis. CompleteMisses specify the number of molecules that do not have to map to any features in the hypothesis. The Misses, FeatureMisses and CompleteMisses were set to 2, 2 and 2, respectively. The minimum inter-feature distance was chosen to be not less than $3.0 \AA$.

\subsubsection{Ligand Pharmacophore Mapping}

The Ligand Pharmacophore Mapping protocol compares a set of ligands to a pharmacophore. A set of 50 synthesized compounds have been mapped against Hypothesis-1 pharmacophore, using the following parameters: Maximum omitted features to be 2 and using Flexible fitting method.

\subsection{Flow Cytometric Analysis of Apoptosis and cell cycle analysis}

The most active compounds as anticancer agent, compounds 6c, 7c, 9c, 10c, 12a, 13a and 14a, were selected as representatives for testing the flow cytometric analysis in two different concentrations each; at $25 \mu \mathrm{g} / \mathrm{mL}$ and at its corresponding $\mathrm{IC}_{50}$ in $\mu \mathrm{g} / \mathrm{mL}$

Flow cytometry was performed with the FACSCalibur system (BD, San Jose, CA, USA). All fluorocytometric data were subsequently analysed and displayed with CELLQUEST software (BD, San Jose, CA, USA). Each analysis included measurements from a minimum of 20000 cells.

After treatment with tested drugs, MCF-7 cells were fixed and permeabilized with $70 \%$ ice-cold ethanol for at least $1 \mathrm{~h}$ and then washed twice in PBS. DNA was stained by incubating the cells at $37^{\circ} \mathrm{C}$ for $1 \mathrm{~h}$ in $40 \mu \mathrm{g} / \mathrm{mL}$ propidium iodide and 100 $\mu \mathrm{g} / \mathrm{mL}$ DNase-free Rnase in PBS. Samples were analyzed by flow cytometry using a FACSCalibur flow cytometer (BDPharmingen). The FL2 red fluorescence channel was assessed on a linear scale, and the percentage of cells undergoing apoptosis was determined as the percentage of hypodiploid cells (sub G0/G1 peak). Dead cells were identified using the Trypan blue exclusion test. 


\section{RESULTS AND DISCUSSION}

\subsection{Chemistry}

The key intermediates, 6-amino-3-benzyl-1H-pyrimidine-2,4-dione 1a\&b, 1-substituted-8-thioxo-3,7,8,9-tetrahydropurine2,6-dione 4a\&b and their derivatives 2-[(1-substituted)-2,6-dioxo-2,3,6,7-tetrahydro-1 $H$-purin-8-ylsulfanyl]- $N$-substituted acetamide 6a-g and 7a-g and 1-substituted-8-[2-(4-substituted phenyl)-2-oxoethylsulfanyl]-3,7-dihydro-1 H-purine-2,6diones $9 \mathrm{a}-\mathrm{g}$ and $10 \mathrm{a}-\mathrm{g}$ were prepared according to reported procedure ${ }^{29}$. The general route to obtain the target $3-(2-$ substituted benzyl)-6-(4-substituted phenyl)-1H-thiazolo[2,3-f]purine-2,4-dione 11a-g and 12a-g and their isosteres $3-$ substituted benzyl-5-methyl-7-substituted-1H-pyrido[2,3-d]pyrimidine-2,4-dione 13a-c and 14a-c is presented in Schemes 1 and 2.

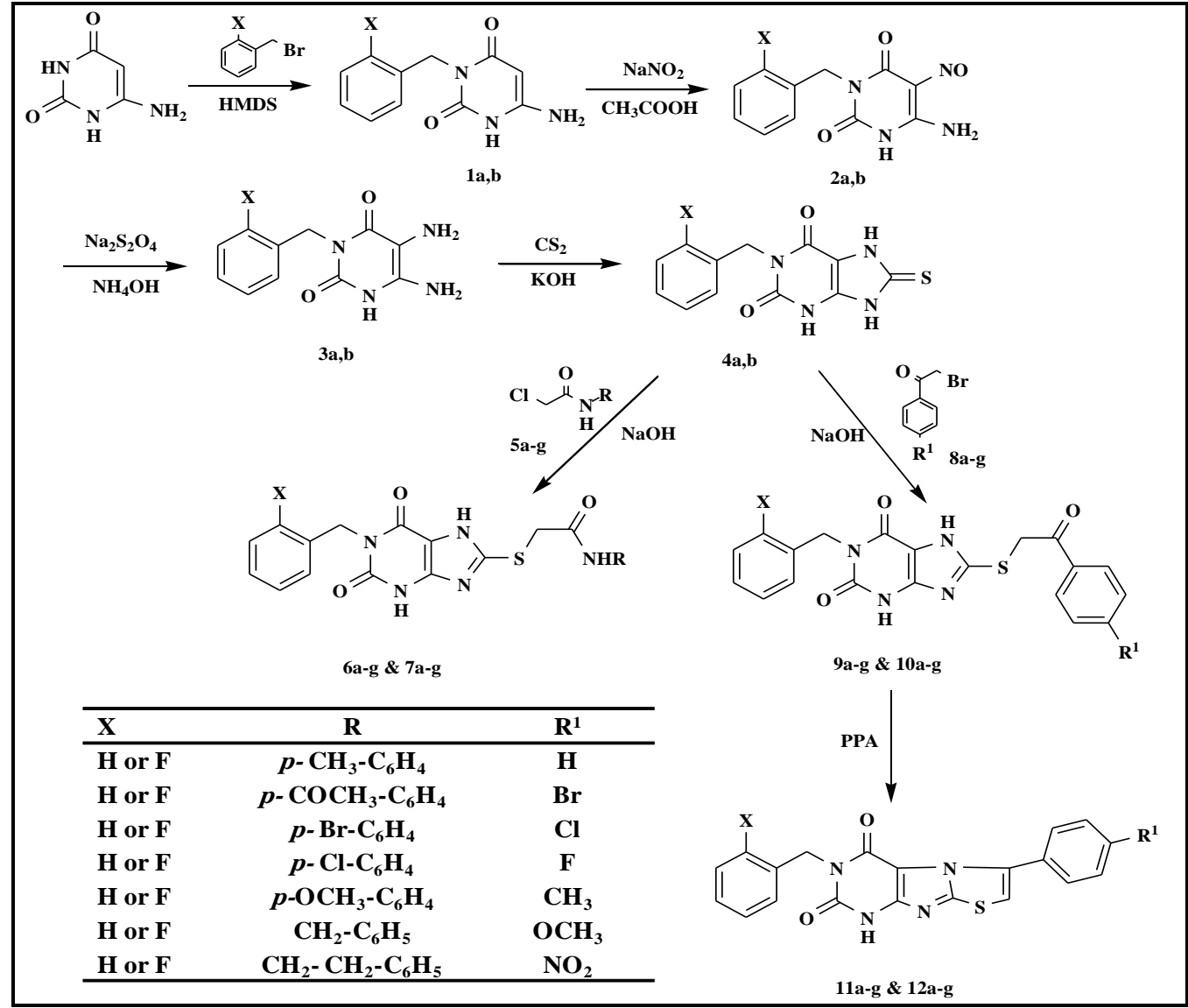

Scheme 1: Synthesis of anilide derivatives 6a-g and 7a-g, phenacyl bromide derivatives 9a-g and 10a-g and their cyclized thiazolo[2,3-f]purine diones 11a-g and 12a-g

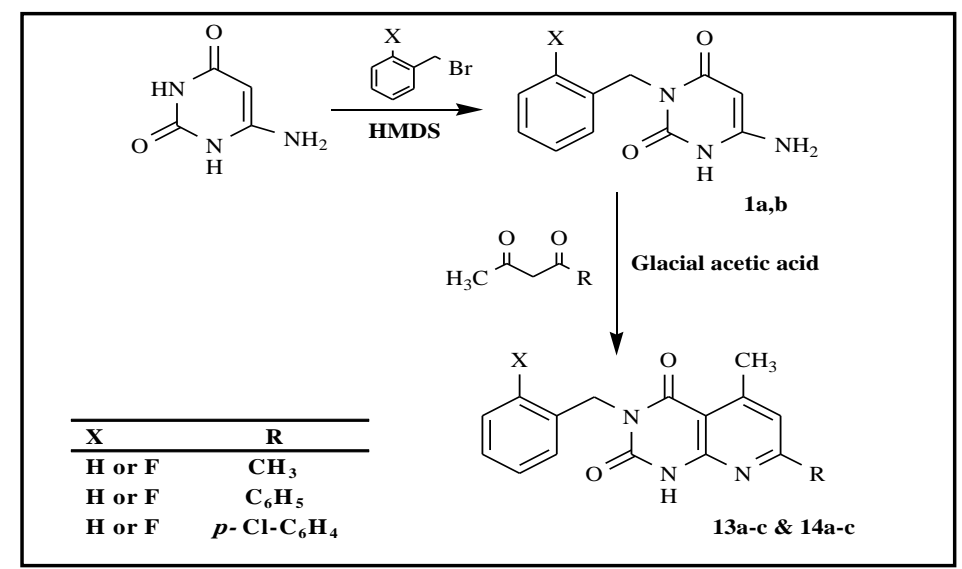

Scheme 2: Synthesis of pyrido[2,3-d]pyrimidine dione derivatives 13a-c and 14a-c 
Trials to use glacial acetic acid or ethanolic solution of hydrochloric acid in cyclodehydration of some 1,8-disubstituted purine-2,6-diones to obtain the required 3,6-disubstituted thiazolo[2,3-d]purine-2,4-diones was reported but did not succeed in our lab ${ }^{16}$. However, considerably better results were obtained through cyclodehydration via polyphosphoric acid (PPA) ${ }^{16,32}$. The advantages of this method were that the yields were considerably high, the method was less timeconsuming, and it was possible to purify the product by recrystallization ${ }^{32}$. Accordingly, cyclodehydration of 1 -substituted8-[2-(4-substituted phenyl)-2-oxoethylsulfanyl]-3,7-dihydro-1H-purine-2,6-diones 9a-g and 10 a-g was carried out in PPA as described in previous works ${ }^{16,32}$ at $130-140^{\circ} \mathrm{C}$ for $5-6 \mathrm{~h}$ (except for 9 f and 10f were heated at $110-120^{\circ} \mathrm{C}$ for $7-8 \mathrm{~h}$ ) afforded the target compounds 3-(2-substituted benzyl)-6-(4-substituted phenyl)-1 H-thiazolo[2,3-f]purine-2,4-dione 11a-g and 12a-g.

Chemical structures of the derivatives $11 \mathrm{a}-\mathrm{g}$ and $12 \mathrm{a}-\mathrm{g}$ were proved by IR, ${ }^{1} \mathbf{H}$ NMR, EI-MS and ESI-HRMS. The purity of the prepared compounds was checked by TLC and further confirmed by elemental analyses. IR spectra of the new compounds 11a-g and 12a-g are characterized by disappearance of N7-H stretching band at $3350-3495 \mathrm{~cm}^{-1}$ which was taken as evidences for cyclization.

${ }^{1} \mathbf{H}$ NMR spectra of compounds 11 a-g and $12 a-g$ showed the $\mathrm{N}_{1}-\mathrm{CH}_{2}$ protons as a singlet at $\delta$ 4.89-5.10 ppm integrating for two protons and $\mathrm{N} 3-\mathrm{H}$ at $\delta 12.00-12.50 \mathrm{ppm}$ which was exchangeable with $\mathrm{D}_{2} \mathrm{O}$. It also showed disappearance of the methylene protons of $\mathrm{S}-\mathrm{CH}_{2}$ and the N7-H proton, in addition to the appearance of $\mathrm{C} 7-\mathrm{H}$ as downfield proton at 7.10-7.40 ppm which are strong evidences for ring cyclization. Some of them were further confirmed by MS (see exp. part), for example, El-MS of compound 11c showed the molecular ion peak $\mathrm{M}^{+}$at $\mathrm{m} / \mathrm{z} 408.05(62.43 \%)$ corresponding to its relative molecular mass (408.04) and a base peak at $\mathrm{m} / \mathrm{z} 91.05(100 \%)$ in addition to $\mathrm{M}^{+}+2$ peak at $\mathrm{m} / \mathrm{z} 410.00(25.32 \%)$, due to ${ }^{37} \mathrm{Cl}$ and the ${ }^{34} \mathrm{~S}$. Also, 12b showed molecular ion peak $\mathrm{M}^{+}$at $\mathrm{m} / \mathrm{z} 469.90(87.15 \%)$ corresponding to its relative molecular mass (469.98), a base peak at $\mathrm{m} / \mathrm{z} 109.00(100 \%)$ in addition to the $\mathrm{M}^{+}+2$ peak at $\mathrm{m} / \mathrm{z} 471.90(77.97 \%)$, due to ${ }^{81} \mathrm{Br}$ and the ${ }^{34} \mathrm{~S}$.

Preparation of 3-substituted benzyl-5-methyl-7-substituted-1H-pyrido[2,3-d]pyrimidine-2,4-dione 13a-c and 14a-c was achieved by condensation of 6-amino-3-(2-substituted benzyl)-1H-pyrimidine-2,4-dione $\mathbf{1 a}$ or $\mathbf{1 b}$ and the appropriate acylacetone derivative in glacial acetic acid through refluxing for 3-4 $\mathrm{h}$.

Although the possibility existed that the condensation might have occurred through the nitrogen of the pyrimidine ring to give pyrimido[1,2-c]pyrimidine $\mathbf{B}$ rather than pyrido[2,3-d] pyrimidine $\mathbf{A}$, this possibility is eliminated, Chart $\mathbf{3}$. If we had structure B for such product, $\mathrm{C} 5-\mathrm{H}$ would appear at $\delta 4.60-4.70 \mathrm{ppm}$ in ${ }^{1} \mathbf{H}$ NMR which disappeared in our case, and this confirms the condensation at $\mathrm{C} 5$ and $\mathrm{N} 6-\mathrm{H}_{2}$. Also, the presence of $\mathrm{N} 1-\mathrm{H}$ around $\delta 10.33-11.99 \mathrm{ppm}$ in ${ }^{1} \mathbf{H} \mathbf{~ N M R}$ confirm this condensation pathway. Further confirmation to eliminate this possibility is proved in our lab by reaction of $1,3-$ disubstituted uracil to block this pathway and gave the pyrido[2,3-d]pyrimidine derivatives ${ }^{33}$.

Chemical structures of the new derivatives 13 a-c and $14 a-c$ were proved by IR, ${ }^{\mathbf{1}} \mathbf{H}$ NMR, EI-MS and ESI-HRMS. The purity of the prepared compounds was checked by TLC and further confirmed by elemental analyses. IR spectra of the new compounds 13a-c and 14a-c are characterized by presence of characteristic bands at $3350-3430 \mathrm{~cm}^{-1}$ of $(\mathrm{N}-\mathrm{H})$ and at $1645-1710 \mathrm{~cm}^{-1}$ of $(\mathrm{C}=\mathrm{O})$ groups. Moreover, they showed the characteristic bands at $3050-3185 \mathrm{~cm}^{-1}(\mathrm{Ar}-\mathrm{H}$ stretching) in addition to its bending at $690-835 \mathrm{~cm}^{-1}$. ${ }^{1} \mathbf{H}$ NMR spectra of compounds 13a-c and 14a-c, showed disappearance of both uracil $\mathrm{C} 5-\mathrm{H}$ at $\delta$ 4.60-4.70 ppm and $\mathrm{N} 6-\mathrm{H}_{2}$ at $\delta 6.17-6.22 \mathrm{ppm}$ and the appearance of $\mathrm{C} 6-\mathrm{H}$ at $\delta 6.63-7.75 \mathrm{ppm}$ and $\mathrm{N} 1-\mathrm{H}$ at $\delta 10.33-11.99 \mathrm{ppm}$ which are strong evidences for ring cyclization. It is also characterized by the appearance of a methyl group at $\delta$ 2.70-3.03 ppm. Also, the structure of representative target compounds 13a-c and 14a-c was confirmed by EI-MS (see experimental part). For example, EI-MS of compound 14a revealed the molecular ion peak $\mathrm{M}^{+}$at $\mathrm{m} / \mathrm{z}$ 299.00 (48.23\%) corresponding to its relative molecular mass (299.11) and a base peak at $\mathrm{m} / \mathrm{z} 109.00(100 \%)$. Also, 14c showed the molecular ion peak $\mathrm{M}^{+}$at $\mathrm{m} / \mathrm{z} 394.90$ (26.90\%) corresponding to its relative molecular mass (395.08) and a base peak at $m / z 109.00(100 \%)$ in addition to $\mathrm{M}^{+}+2$ peak at $\mathrm{m} / z 396.90(10.13 \%)$, due to ${ }^{37} \mathrm{Cl}$.<smiles></smiles>

Chart 3; The possible cyclization pathways

\subsection{Anticancer activity:}

The target compounds $\mathbf{4 a}, \mathbf{4 b}, \mathbf{6 c}, \mathbf{7 c}, \mathbf{9 c}, \mathbf{1 0 a}, \mathbf{1 0 c}, \mathbf{1 2 a}, \mathbf{1 3 a}, \mathbf{1 3 b}$ and $\mathbf{1 4 a}$ were chosen to test for their anticancer activity against human breast cancer cell line MCF-7 in comparison to Doxorubicin (DOX) using the Sulphorhodamine-B (SRB) assay, after $72 \mathrm{~h}$ exposure ${ }^{30}$. The data were fitted to sigmoidal concentration response curves and the corresponding $\mathrm{IC}_{50}$ values were calculated using commercially available software (GraphPad Prizm). Most of the tested compounds showed good to excellent inhibition activity against the tested cell line in comparison to DOX, Table 1. It was noticed that compounds $7 c(X=F, R=B r)$ and $9 c\left(X=H, R^{1}=C l\right)$ were the most potent cytotoxic agents with $I_{50}$ value of $8.87,7.25$ $\mu \mathrm{mol} / \mathrm{mL}$ respectively $\left(\mathrm{IC}_{50}\right.$ of $\mathrm{DOX}$ is $\left.7.73 \mu \mathrm{mol} / \mathrm{mL}\right)$. 
Moreover, compounds 10c $\left(X=F, R^{1}=C l\right), 12 a\left(X=F, R^{1}=H\right), 13 a\left(X=H, R=C_{3}\right)$ and 13b $\left(X=H, R=C_{6} H_{5}\right)$ showed good cytotoxic activity compared to DOX as they showed $\mathrm{IC}_{50}$ value of $10.04,14.00,13.41$ and $11.98 \mu \mathrm{mol} / \mathrm{mL}$ respectively ( $\mathrm{IC}_{50}$ of Doxorubicin is $7.73 \mu \mathrm{mol} / \mathrm{mL}$ ).

From these data, it was concluded that (1) The phenacyl bromide derivative $9 c\left(X=H, R^{1}=\mathrm{Cl}\right.$, with $I C_{50}$ of $\left.7.25 \mu \mathrm{mol} / \mathrm{mL}\right)$, was more active than the anilide derivative $7 c\left(X=F, R=B r\right.$, with $I_{50}$ of $\left.8.87 \mu \mathrm{mol} / \mathrm{mL}\right)$. (2) Cyclization of the phenacyl bromide derivative $10 \mathrm{a}\left(\mathrm{X}=\mathrm{F}, \mathrm{R}^{1}=\mathrm{H}\right.$, with $\mathrm{IC}_{50}$ of $\left.56.82 \mu \mathrm{mol} / \mathrm{mL}\right)$ into the corresponding thiazolopurine 12a (with $\mathrm{IC}_{50}$ of $14.00 \mu \mathrm{mol} / \mathrm{mL}$ ) emphasizes the importance of cyclization and addition of thiazole ring for anticancer activity. (3) Results also showed the significance of pyridopyrimidines $13 \mathbf{a}\left(\mathrm{X}=\mathrm{H}, \mathrm{R}=\mathrm{CH}_{3}\right)$ and $\mathbf{1 3 b}\left(\mathrm{X}=\mathrm{H}, \mathrm{R}=\mathrm{C}_{6} \mathrm{H}_{5}\right)$ as anticancer agents as they were potent cytotoxic agent. (4) Benzyl derivatives are more active than the fluorobenzyl derivatives as in some cases the insertion of the fluoro substituent decreased the activity, such as: $4 a\left(X=H, I C_{50}\right.$ of $\left.15.64 \mu \mathrm{mol} / \mathrm{mL}\right) \mathrm{compared}$ to $4 \mathrm{~b}\left(X=\mathrm{F}, \mathrm{IC}_{50}\right.$ of $\left.19.95 \mu \mathrm{mol} / \mathrm{mL}\right)$, also, $9 \mathrm{c}\left(X=\mathrm{H}, \mathrm{R}^{1}=\mathrm{Cl}, \mathrm{IC}_{50}\right.$ of $\left.7.25 \mu \mathrm{mol} / \mathrm{mL}\right)$ compared to $10 \mathrm{c}\left(X=\mathrm{F}, \mathrm{R}^{1}=\mathrm{Cl}, \mathrm{IC}_{50}\right.$ of $10.04 \mu \mathrm{mol} / \mathrm{mL})$ and $13 \mathrm{a}\left(\mathrm{X}=\mathrm{H}, \mathrm{R}=\mathrm{CH}_{3}, \mathrm{IC}_{50}\right.$ of $\left.13.41 \mu \mathrm{mol} / \mathrm{mL}\right)$ compared to $14 \mathrm{a}\left(X=\mathrm{F}, \mathrm{R}=\mathrm{CH}_{3}, \mathrm{IC}_{50}\right.$ of $\left.69.5 \mu \mathrm{mol} / \mathrm{mL}\right)$. In other cases, the introduction of fluoro substituent increased the activity as shown in $6 c\left(X=H, R=B r, I C_{50}\right.$ of 18.35 $\mu \mathrm{mol} / \mathrm{mL})$ and $7 \mathrm{c}\left(X=F, R=B r, I C_{50}\right.$ of $\left.8.87 \mu \mathrm{mol} / \mathrm{mL}\right)$.

\subsection{Molecular Modeling Study}

\subsubsection{HipHop Pharmacophore}

Accelrys Discovery Studio Software package (DS) was used for automated ligand-based pharmacophore generation HipHop is intended to derive common feature pharmacophore models using information from a set of active compounds. The retrieved pharmacophore models are expected to discriminate between active and inactive compounds. HipHop identifies configurations or three-dimensional spatial arrangements of chemical features that are common to molecules in a training set. The configurations are identified by a pruned exhaustive search, starting with small sets of features and extending them until no larger common configuration is found. The resultant pharmacophores are ranked as they are built. The ranking is a measure of how well the molecules map onto the proposed pharmacophores, as well as the rarity of the pharmacophore model. If a pharmacophore model is less likely to map to an inactive compound, it will be given a higher rank ${ }^{34-36}$. In our study, we focused on ligand-based virtual screening techniques to identify novel compounds with activity for CDK1 inhibitors. We started the development of ligand-based pharmacophore models based on training set compounds with high activity $\left(\mathrm{IC}_{50}<1000 \mathrm{nM}\right)$ and several-fold selectivity for CDK1. Afterward, the model was carefully and iteratively refined with exclusion volume spheres (XVOLs) and shape constraints. The final model was used to virtually screen in house database. In order to implement this modeling work-flow, a dataset of 30 active and 15 biologically inactive compounds was collected from the literature based on chemical structure and CDK1 in vitro biological diversity, Table $2^{37-50}$.

Table 2: Data set of 30 training set of CDK1 inhibitors

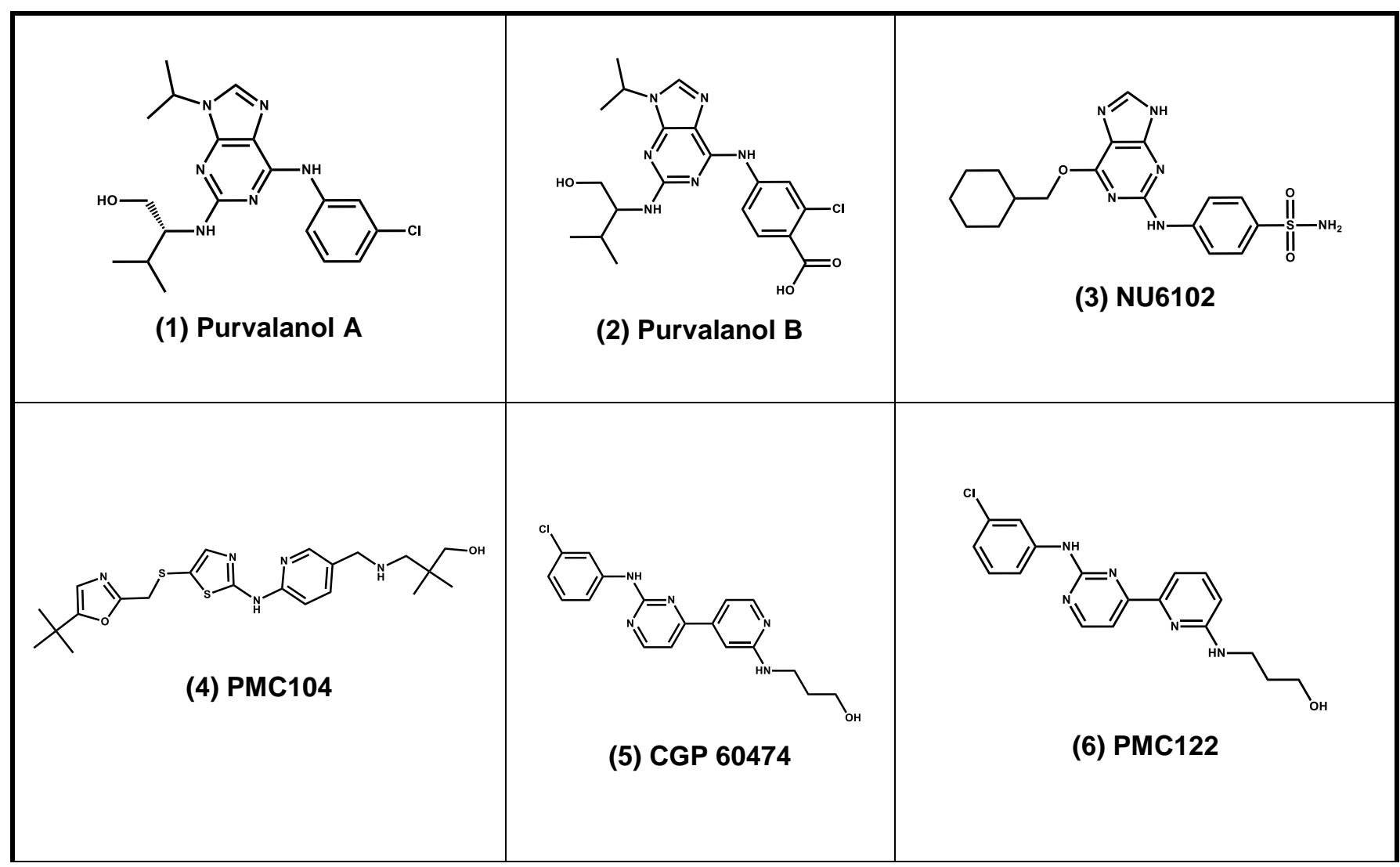




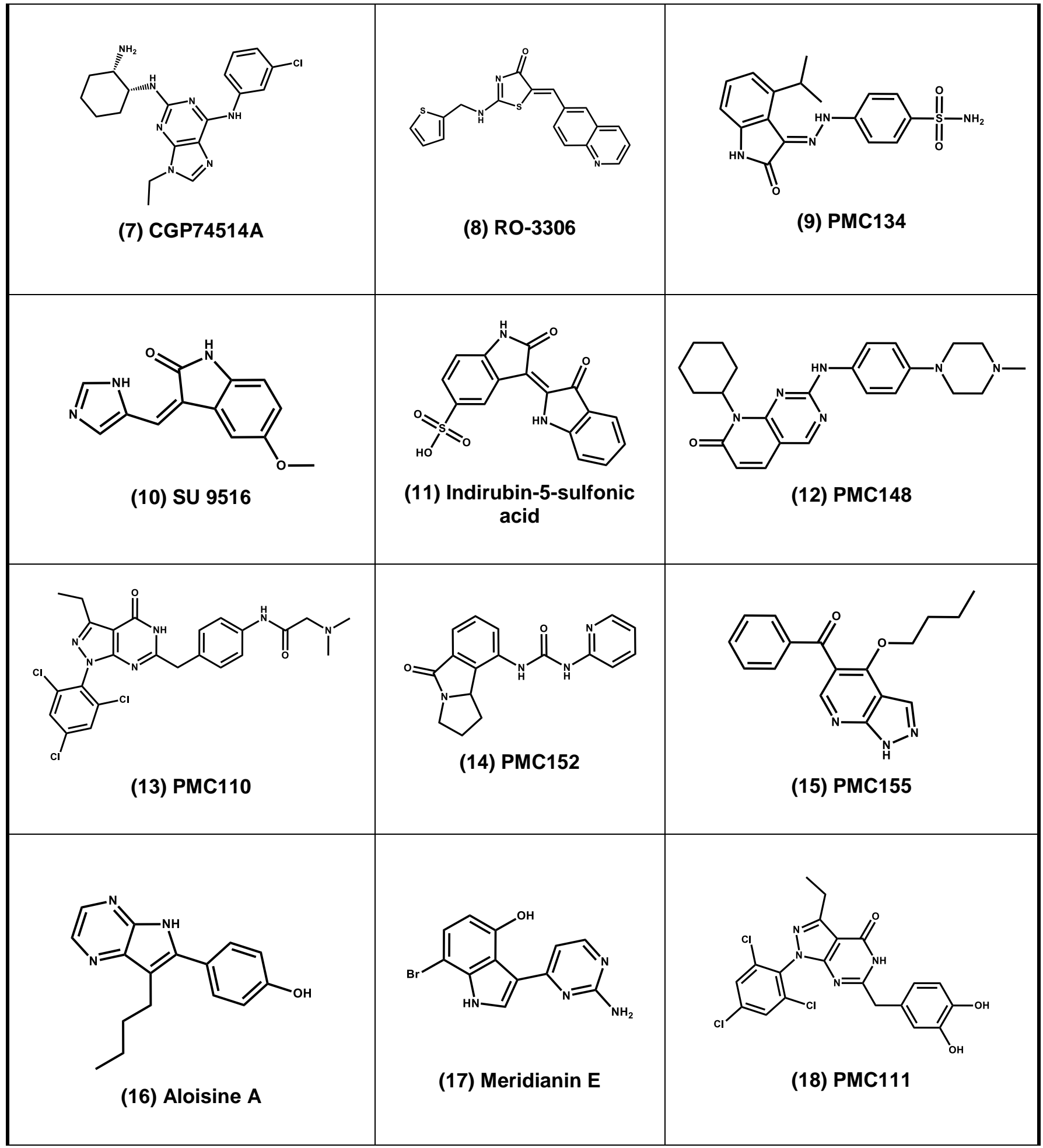




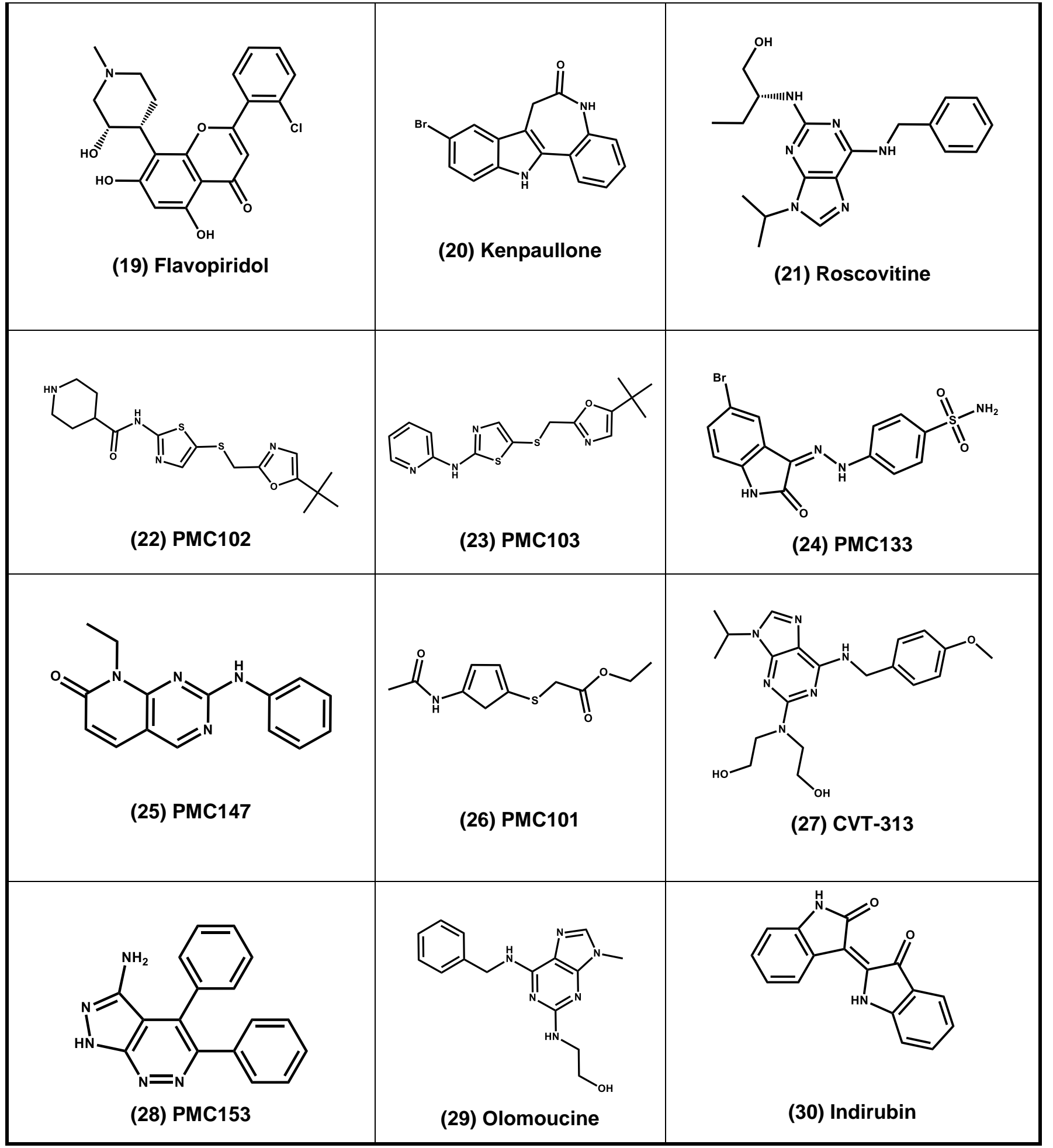

In order to generate the best model, a lot of HipHop runs were carried out by varying the control parameters. In the model generation methodology, the highest weighing was assigned to the most active compound in the training set (Purvalanol $\mathrm{A} ; \mathrm{IC}_{50} 1 \mu \mathrm{M}$ ), this was achieved by putting 2 (which ensures that all of the chemical features in the compound will be considered in building hypotheses space) and 0 (which forces mapping of all features of compound) in principle and maximum omitting features columns, respectively. For the moderately active compounds $\left(\mathrm{IC}_{50}<10 \mathrm{nM}\right)$, the value of 1 (ensures that at least one mapping for each of generated hypotheses will be found) and 1 (all but one feature must map) were put in principle and maximum omitting feature column respectively. For the lower active compounds the value of 0 (indicates that the molecule is inactive and will not be used in the creation of the pharmacophore) and 1 were put in principle and maximum omitting feature column respectively. Feature Mapping protocol was used to identify the common chemical groups present in the training set compounds. As predicted, hydrogen bond acceptor (HBA), Hydrogen Bond 
Donor (HBD), Hydrophobic (HY), and Ring_Aromatic (RA), Positive_lonizable, Negative_lonizable features were selected during the pharmacophore generation.

The successful HipHop run resulted in the generation of 10 hypotheses, each composed of four features, The 10 hypotheses (Hypos) generated had scores from 173.292 to 166.657 , Table 3.

This small range of ranking score suggests that the features are spatially arranged in a similar fashion in all 10 models. To determine the similarity between the ten hypotheses, a hierarchical cluster analysis was performed. The results of the cluster analysis indicate that hypos 1 and 10 belong to the same cluster, whereas hypos 2, 3, 4, 5, 6, 7, 8 and 9 are slightly different. All ten hypotheses contain four features, of which one ring aromatic (RA) feature and one Hydrophobic (HY) are common for all except Hypo 6 which has no RA feature. The first two hypotheses show close statistics in terms of their rank score which is quite significantly better than the rest hypotheses. Consequently, both hypotheses (Hypo-1 and Hypo-2) were considered for further analysis.

Table 3: Summary of HipHop generated common feature hypothesis:

\begin{tabular}{|c|c|c|c|c|}
\hline Hypothesis & $\begin{array}{c}\text { Features } \\
(\text { a) }\end{array}$ & $\begin{array}{c}\text { Rank } \\
\text { Score }\end{array}$ & Direct Hit $^{(\mathrm{b})}$ & Partial Hit $^{(\mathrm{c})}$ \\
\hline \hline 01 & RHHA & 173.292 & 011111111111110111101111 & 100000000000001000010000 \\
\hline 02 & RRHA & 173.126 & 1111110011111101111111111 & 000000110000001000000000 \\
\hline 03 & RRHA & 171.971 & 111111011011110111111111 & 000000100100001000000000 \\
\hline 04 & RRHA & 171.971 & 111111011011110111111111 & 000000100100001000000000 \\
\hline 05 & RRHA & 170.761 & 1111110110111101111111111 & 000000100100001000000000 \\
\hline 06 & HHDA & 170.656 & 010111111111110111111111 & 101000000000001000000000 \\
\hline 07 & RHAA & 168.541 & 1101110111111110111111111 & 001000100000001000000000 \\
\hline 08 & RHAA & 168.068 & 110111011111110111111111 & 001000100000001000000000 \\
\hline 09 & RHAA & 166.659 & 11011101111111101111111111 & 001000100000001000000000 \\
\hline 10 & RHHA & 166.657 & 011111011111110111111111 & 100000100000001000000000 \\
\hline
\end{tabular}

(a) R, Ring Aromatic; H, hydrophobic; A, hydrogen bond acceptor.

(b) Direct hit indicates whether (1) or not (0) a molecule in the training set mapped to every feature in the hypothesis.

(c) Partial hit indicates whether (1) or not (0) a molecule in the training set mapped to all but one feature in the hypothesis.

\subsubsection{Analysis of Hypo-1 and Hypo-2}

Examination of Hypo-1, Fig. 1,A shows two hydrophobic features (HY-1 and HY-2), a hydrogen bond acceptor (HBA) and a ring aromatic feature (RA). The two hydrophobic features are apart from each other by inter-feature distance of $4.5 \AA$. As for the Hypo-2, Fig. 1,B, the four features are somewhat different showing one hydrophobic feature (HY-1) and two ring aromatic features (RA-1 and RA-2) and with a hydrogen bond acceptor feature. The tested compounds were mapped onto Hypo-1 and Hypo-2 using the "best fit" option where HipHop scores the orientation of a mapped compound within the hypothesis features using a "fit value" score. For models that show four features, as in this case, the maximum fit value score is 4.0 .

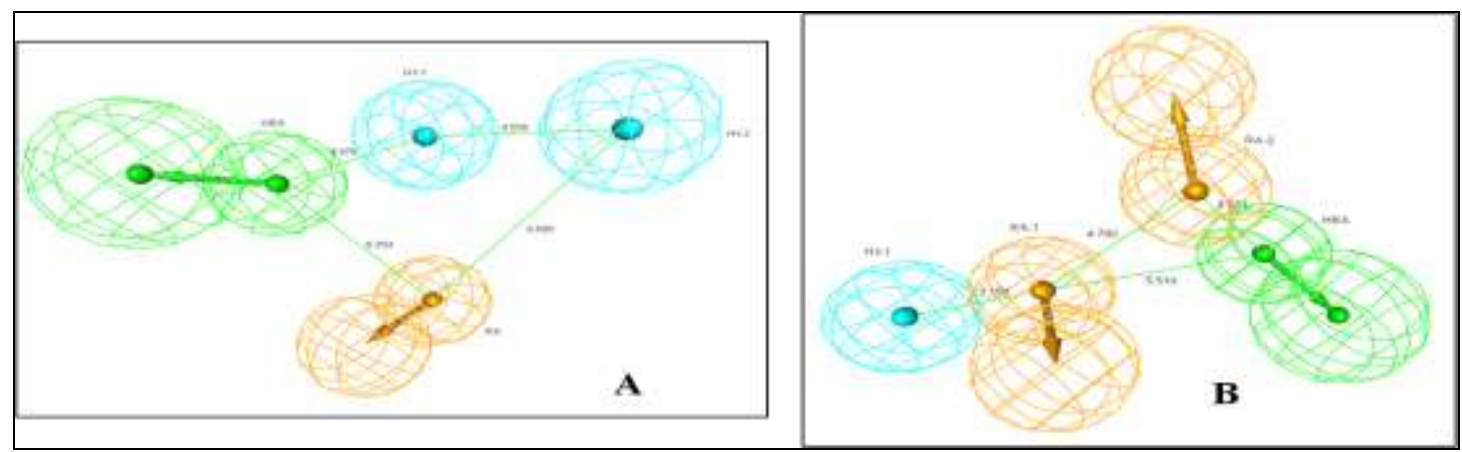

Figure 1,A: Hypo-1 showing inter-feature distances $(\AA)$, B: Hypo-2 showing inter-feature distances $(\AA ̊)$. 
Features are colour coded as: Hydrophobic, cyan; Hydrogen bond acceptor, green, Ring Aromatic, orange.

Results from the mapped compounds, Table 4 showed a rough correlation between the fit values and their biological activities. The most active compound (Purvalanol A) was assigned the highest fit value by both models. However both models can not differentiate between the small differences in biological activity exhibited by the rest of the active compounds. Mapping of compound (Purvalanol A) to both models (Hypo-1 and Hypo-2) is shown in Fig. 2.

Table 4: Output for Hypo-1 and Hypo-2 mapping

\begin{tabular}{|c|c|c|c|c|c|}
\hline \multirow{2}{*}{ Compound } & \multirow{2}{*}{$\mathrm{IC}_{50}(\mathrm{nM})$} & \multicolumn{2}{|l|}{ Fit value } & \multicolumn{2}{|c|}{$E(\mathrm{kcal} / \mathrm{mol})^{\mathrm{a}}$} \\
\hline & & Hyp-1 & Hyp-2 & Hyp-1 & Hyp-2 \\
\hline Purvalanol A & 0.0040 & 3.99982 & 3.99990 & 18.15480 & 19.43690 \\
\hline Purvalanol B & 0.0060 & 3.43070 & 3.68802 & 5.38114 & 10.32610 \\
\hline NU6102 & 0.0095 & 2.64262 & 0.76285 & 4.43556 & 13.03910 \\
\hline PMC104 & 0.0180 & 2.57900 & 3.05038 & 10.78220 & 19.98450 \\
\hline CGP 60474 & 0.0200 & 3.20182 & 3.32401 & 2.34222 & 14.29670 \\
\hline PMC122 & 0.0200 & 2.99200 & 2.82664 & 18.28480 & 10.72110 \\
\hline CGP74514A & 0.0250 & 2.23308 & 2.16324 & 11.80130 & 15.54800 \\
\hline RO-3306 & 0.0350 & 2.75914 & 3.06869 & 13.46750 & 8.71847 \\
\hline PMC134 & 0.0370 & 1.88601 & 2.53830 & 15.88930 & 18.65870 \\
\hline SU 9516 & 0.0400 & 2.37105 & 2.86794 & 1.27289 & 0.03687 \\
\hline Indirubin-5-sulfonic acid & 0.0550 & 2.35306 & 1.97079 & 0.13574 & 0.03119 \\
\hline PMC148 & 0.0790 & 3.09696 & 1.06825 & 11.16860 & 10.92790 \\
\hline PMC110 & 0.1000 & 2.65747 & 2.45176 & 12.36690 & 18.14150 \\
\hline PMC152 & 0.1200 & 1.05235 & 0.78202 & 1.60350 & 1.59593 \\
\hline PMC155 & 0.1500 & 2.70099 & 1.75875 & 1.49593 & 2.64313 \\
\hline Aloisine A & 0.1500 & 1.00974 & 0.59599 & 0.03732 & 0.76521 \\
\hline Meridianin E & 0.1800 & 2.54131 & 2.32808 & 3.70928 & 0.15680 \\
\hline PMC111 & 0.2400 & 2.39782 & 2.26169 & 2.07870 & 13.63680 \\
\hline Flavopiridol & 0.3000 & 3.05840 & 2.47295 & 14.60780 & 18.90930 \\
\hline Kenpaullone & 0.4000 & 0.12510 & 1.54723 & 0.00162 & 0.00162 \\
\hline Roscovitine & 0.4500 & 3.24415 & 3.00109 & 7.94801 & 8.75622 \\
\hline PMC102 & 0.4800 & 2.55044 & 2.60564 & 6.96686 & 2.56536 \\
\hline PMC103 & 0.4800 & 2.33536 & 2.41849 & 7.66487 & 11.17070 \\
\hline PMC133 & 0.7800 & 2.72069 & 2.53458 & 2.52811 & 15.85060 \\
\hline PMC147 & 1.0000 & 1.13752 & 1.27160 & 0.00000 & 0.00000 \\
\hline PMC101 & 1.9000 & 2.86885 & 1.99229 & 13.10650 & 3.04452 \\
\hline CVT-313 & 4.2000 & 2.02903 & 3.03385 & 2.45691 & 7.17578 \\
\hline PMC153 & 6.0000 & 2.19827 & 1.02872 & .505543 & 0.00000 \\
\hline Olomoucine & 7.0000 & 2.64728 & 3.22910 & 7.75201 & 7.75201 \\
\hline Indirubin & 10.0000 & 2.31198 & 1.49944 & 0.00000 & 0.00000 \\
\hline
\end{tabular}

(a) Relative energy of the fitted conformer. 


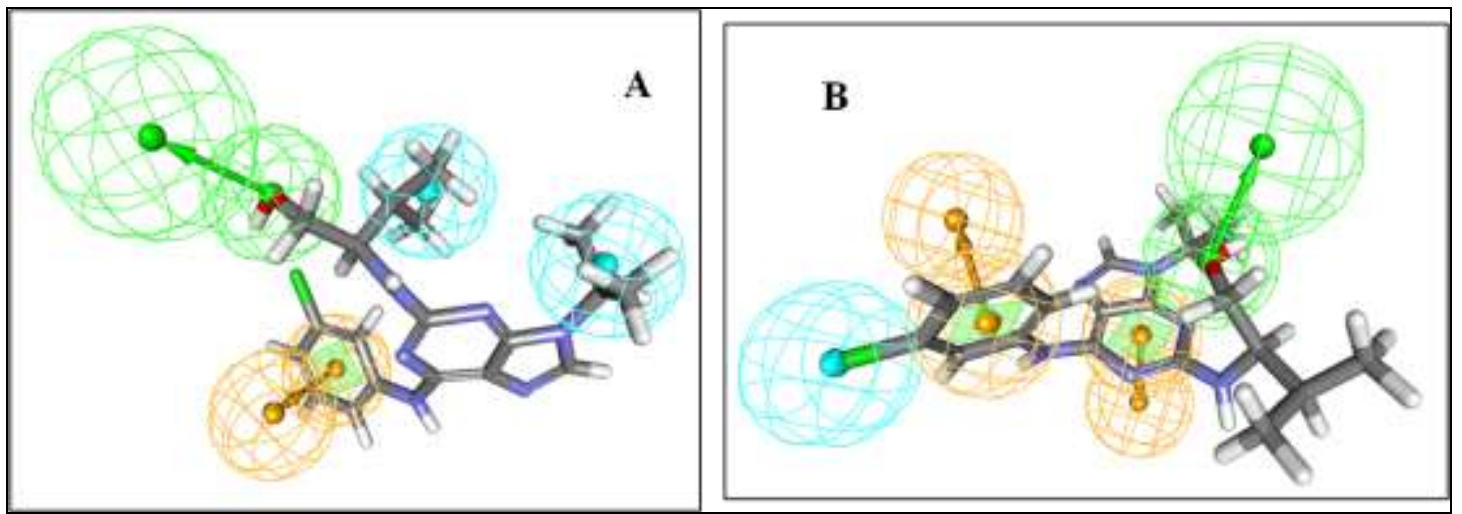

Figure 2. Mapping of (Purvalanol A) (stick) to (A) Hypo-1 and (B) Hypo-2.

Mapping of all compounds reveals the predominance of Hypo-1 over Hypo-2 in mapping of the training set and also showed the importance of presence of certain groups to fit the produced model. The two hydrophobic features (HY-1 and HY-2) can accommodate either an aromatic ring, cycloalkyl, alkyl and halogen groups. The hydrogen bond acceptor feature (HBA) is occupied by carbonyl, sulfonyl or heteroatom within aromatic ring. Ring aromatic features (RA-1) accommodate five or six membered aromatic rings.

\subsubsection{Screening of in-house dataset of newly synthesized compounds:}

Mapping the dataset of the 50 synthesized compounds to Hypo-1 using Ligand Pharmacophore Mapping Protocol was done to investigate the activity of the synthesized Ligands against CDK1, as anticancer candidates shown that of the 50 compounds, 46 compounds mapped to the Pharmacophore Model with different fit values. The most active compound (9c) in the anticancer assay showed the best fit value, Fig. 3, however there was a rough correlation between the anticancer activity and the rest of the tested compounds, Table 5. Generally, theoretical suggestion is comparable to some extent with the practical one.

Table 5: Fit value for the tested synthesized compounds against Hypo-1 together with anti-proliferative activity against

MCF-7 as IC $_{50}$

\begin{tabular}{|c|c|c|}
\hline Compound & $\mathbf{I C}_{50}(\mu \mathrm{mol} / \mathrm{mL})$ & Fit value \\
\hline \hline 9c & 7.25 & 3.57403 \\
\hline $\mathbf{7 c}$ & 8.87 & 3.48897 \\
\hline $\mathbf{1 0 c}$ & 10.04 & 2.98574 \\
\hline $\mathbf{1 3 b}$ & 11.98 & 2.76242 \\
\hline $\mathbf{1 3 a}$ & 13.41 & 2.75817 \\
\hline $\mathbf{1 2 a}$ & 14.00 & 2.79434 \\
\hline $\mathbf{4 a}$ & 15.64 & 2.39551 \\
\hline $\mathbf{6 c}$ & 18.35 & 3.36915 \\
\hline $\mathbf{4 b}$ & 19.95 & 2.36067 \\
\hline $\mathbf{1 0 a}$ & 56.82 & 2.87789 \\
\hline $\mathbf{1 4 a}$ & 69.54 & 2.77761 \\
\hline & & \\
\hline & & \\
\hline
\end{tabular}




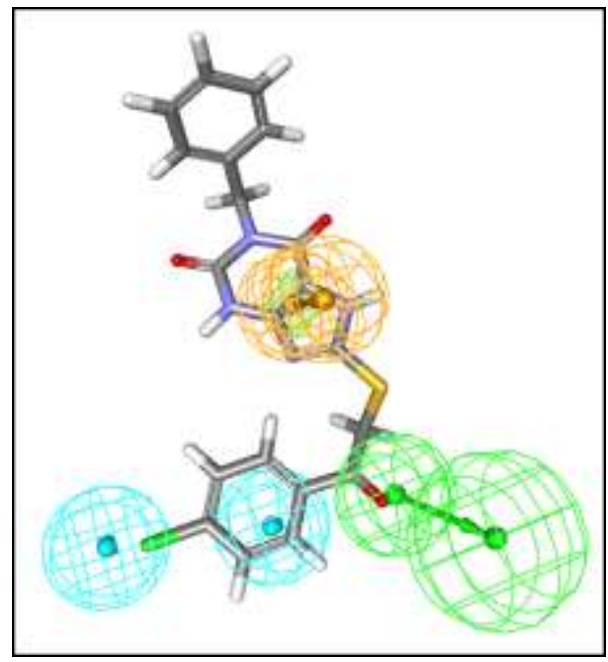

Figure 3. Mapping of (9c) (stick) to Hypo-1.

\subsection{Flow Cytometric Analysis}

On the basis of the in-vitro cytotoxicity data, the most potent compounds $6 \mathrm{c}, 7 \mathrm{c}, 9 \mathrm{c}, 10 \mathrm{c}, 12 \mathrm{a}, 13 \mathrm{a}$ and 14a against MCF-7 cells line were selected for further mechanistic study. MCF-7 cells were not only treated with DMSO (0.1\%) alone as controls, but were treated with two concentrations of each tested compounds (at $25 \mu \mathrm{mol} / \mathrm{mL}$ and at each ones's corresponding $\mathrm{IC}_{50}$ in $\mu \mathrm{mol} / \mathrm{mL}$ ) for $24 \mathrm{~h}$ and stained with propidium iodide (PI). The effects on cell cycle distribution of MCF-7 cells were evaluated by flow cytometry. The inhibition of cancer cell proliferation, the cessation of cell cycle progression, and the induction of apoptosis have all been targeted in chemotherapeutic strategies for the treatment of cancer. We therefore evaluated whether tested compounds alter the cell cycle of MCF-7 cells using PI and flow cytometric analyses, Table 6. Figure 4 shows representative experiment; we found that the percentage of apoptotic cells was comparable to $3 \%$ in the untreated cells. Treatment with tested compounds showed $27.74-51.60 \%$ as percentage of apoptotic cells at the $\mathrm{IC}_{50}$ of tested compounds, and from $33.90-47.99 \%$ of apoptotic cells at a concentration of 25 $\mu \mathrm{mol} / \mathrm{mL}$. This increased induction of apoptosis after treatment with tested compounds was inversely correlated with a significant decrease in the percentage of cells in S phase. The total data from different experiments $(n=70$ for MCF-7 cells) demonstrated that treatment with tested compounds significantly potentiated apoptosis in MCF-7 cells (Table 6). It can be seen that there is no great difference in induction of apoptosis between the two concentrations of the tested compounds either at the $\mathrm{IC}_{50}$ or at a concentration of $25 \mu \mathrm{mol} / \mathrm{mL}$ which eliminate the importance of high dosage of the compounds to prevent toxicity.

Table 6: Flowcytometric analysis

\begin{tabular}{|c|c|c|}
\hline Compound & $\begin{array}{c}\text { Apoptosis \% at } \\
\text { corresponding } \\
\text { IC }_{50}\end{array}$ & $\begin{array}{c}\text { Apoptosis \% at 25 } \\
\mu \mathrm{mol} / \mathrm{mL}\end{array}$ \\
\hline \hline 6c & $\begin{array}{c}40.74 \% \text { at } 18.35 \\
\mu \mathrm{mol} / \mathrm{mL}\end{array}$ & $45.16 \%$ \\
\hline 7c & $\begin{array}{c}51.60 \% \text { at } 8.87 \\
\mu \mathrm{mol} / \mathrm{mL}\end{array}$ & $45.68 \%$ \\
\hline 9c & $\begin{array}{c}42.32 \% \text { at } 7.25 \\
\mu \mathrm{mol} / \mathrm{mL}\end{array}$ & $41.80 \%$ \\
\hline 10c & $\begin{array}{c}51.59 \% \text { at } 10.04 \\
\mu \mathrm{mol} / \mathrm{mL}\end{array}$ & $45.36 \%$ \\
\hline 12a & $\begin{array}{c}49.61 \% \text { at } 14.00 \\
\mu \mathrm{mol} / \mathrm{mL}\end{array}$ & $47.99 \%$ \\
\hline 13a & $\begin{array}{c}34.31 \% \text { at } 13.41 \\
\mu \mathrm{mol} / \mathrm{mL}\end{array}$ & $33.90 \%$ \\
\hline 14a & $\begin{array}{c}27.74 \% \text { at } 69.54 \\
\mu \mathrm{mol} / \mathrm{mL}\end{array}$ & $37.24 \%$ \\
\hline
\end{tabular}




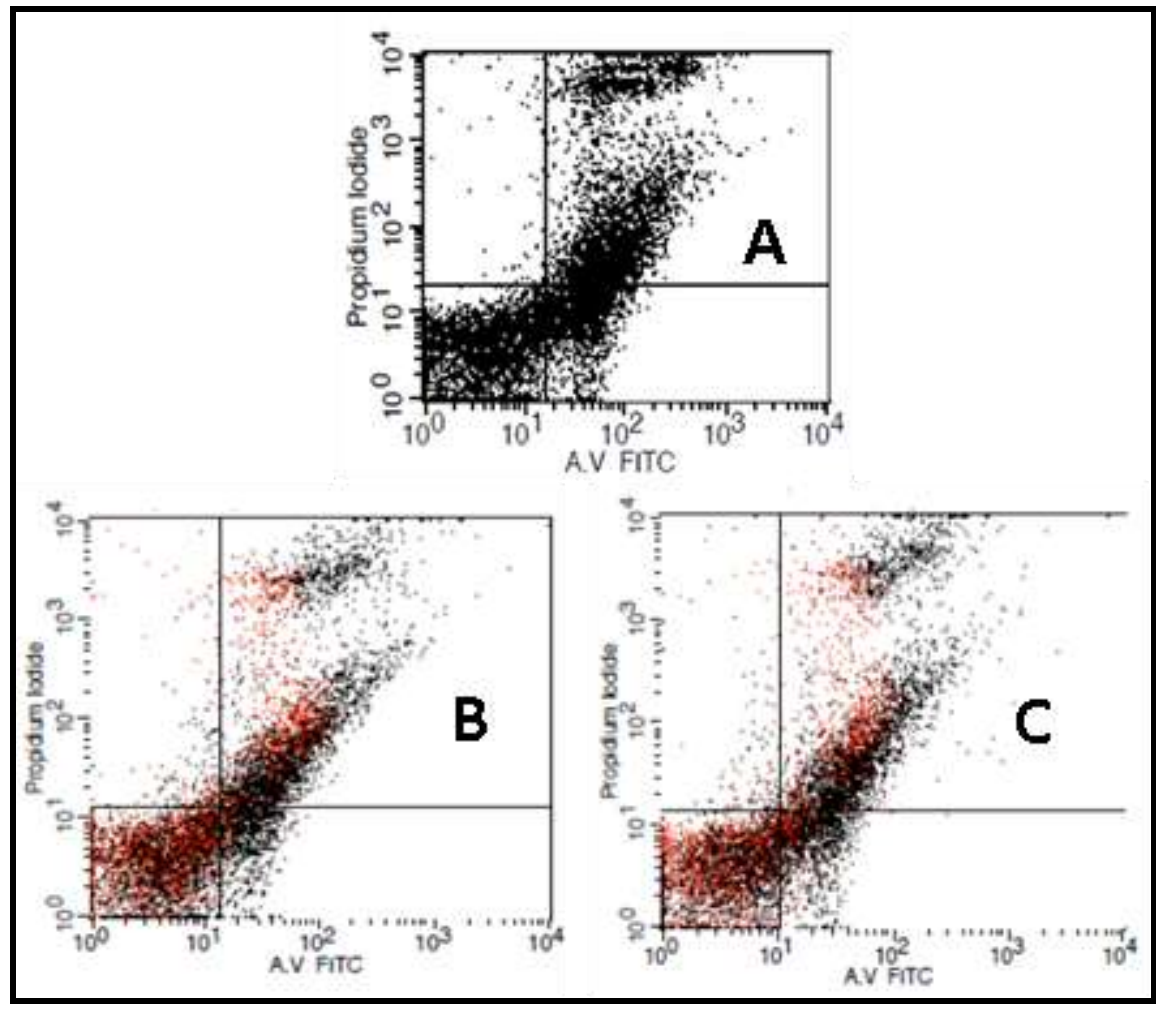

Figure 4, The ability of representative compounds to alter cell cycle;

A: DMSO, B: 13a at $25 \mu \mathrm{mol} / \mathrm{mL}, \mathrm{C}: 13 \mathrm{a}$ at its $\mathrm{IC}_{50}(13.41 \mu \mathrm{mol} / \mathrm{mL})$

\section{CONCLUSION}

In the present work, the synthesis of thiazolo[2,3-f]purine-2,4-diones $11 \mathbf{a - g}$ and $12 \mathbf{a}-\mathbf{g}$ and their isosteres pyrido[2,3d]pyrimidine-2,4-diones 13a-c and 14a-c were designed and synthesized. The proposed structures of the new compounds were confirmed by spectroscopic and elemental analyses. The anticancer activity of most of the newly synthesized compounds showed good to excellent inhibition activity against the tested human breast cancer cell line MCF-7 in comparison to doxorubicin as a reference drug.

\section{REFERENCES:}

1. Mullaney, I., An introduction to anticancer drugs. Pharmacology in One Semester 2012, chapter 24.

2. Palop, J. A.; Plano, D.; Moreno, E.; Sanmartín, C., Novel quinazoline and pyrido[2,3-d]pyrimidine derivatives and their hydroselenite salts as antitumoral agents. ARKIVOC 2014, 2, 187-206.

3. Moreno, E.; Plano, D.; Lamberto, I.; Font, M.; Encío, I.; Palop, J. A.; Sanmartín, C., Sulfur and selenium derivatives of quinazoline and pyrido[2, 3-d]pyrimidine: Synthesis and study of their potential cytotoxic activity in vitro. Eur. J. Med. Chem. 2012, 47, 283-298.

4. Haesslein, J.; Jullian, N., Recent advances in cyclin-dependent kinase inhibition. Purine-based derivatives as anti-cancer agents. Roles and perspectives for the future. Curr. Top. Med. Chem. 2002, 2 (9), 1037-1050.

5. Legraverend, M.; Tunnah, P.; Noble, M.; Ducrot, P.; Ludwig, O.; Grierson, D. S.; Leost, M.; Meijer, L.; Endicott, J., Cyclin-dependent kinase inhibition by new C-2 alkynylated purine derivatives and molecular structure of a CDK2 inhibitor complex. J. Med. Chem. 2000, 43 (7), 1282-1292.

6. Azevedo, W. F.; Leclerc, S.; Meijer, L.; Havlicek, L.; Strnad, M.; Kim, S. H., Inhibition of Cyclin-Dependent Kinases by Purine Analogues. Eur. J. Biochem. 1997, 243 (1-2), 518-526.

7. Kryštof, V. r.; Lenobel, R.; Havlíček, L.; Kuzma, M.; Strnad, M., Synthesis and biological activity of olomoucine II. Bioorg. Med. Chem. Lett. 2002, 12 (22), 3283-3286.

8. Weyler, S.; Hayallah, A. M.; Müller, C. E., Versatile, convenient synthesis of pyrimido[1,2,3-cd]purinediones. Tetrahedron 2003, 59 (1), 47-54.

9. Sadzuka, Y.; Sugiyama, T.; Suzuki, H.; Sawanishi, H.; Miyamoto, K.-i., Increased effects of MPDAX, a novel xanthine derivative, on antitumor activity of doxorubicin. Toxicol. Lett. 2004, 150 (3), 341-349.

10. Hayallah, A. M.; Momekov, G.; Famulok, M., Antitumor activity of some new 1,3,8-trisubstituted purine-2,6-diones and 1,3,6-trisubstituted thiazolo[2,3-f]purine-2,4-diones. Bull. Pharm. Sci., Assiut University 2008, 31, $391-399$. 
11. Kode, N. R.; Phadtare, S., Synthesis and cytotoxic activity of some new 2,6-substituted purines. Molecules 2011, 16 (7), 5840-5860.

12. Ashour, F. A.; Rida, S. M.; El-Hawash, S. A.; EISemary, M. M.; Badr, M. H., Synthesis, anticancer, anti-HIV-1, and antimicrobial activity of some tricyclic triazino and triazolo[4,3-e]purine derivatives. Med. Chem. Res. 2012,21 (7), 1107-1119.

13. Ibrahim, D. A.; Ismail, N. S., Design, synthesis and biological study of novel pyrido[2,3-d]pyrimidine as antiproliferative CDK2 inhibitors. Eur. J. Med. Chem. 2011, 46 (12), 5825-5832.

14. Shanmugasundaram, P.; Harikrishnan, N.; Vijey Aanandini, M.; Sathish Kumar, M.; Sateesh, J., Synthesis and biological evaluation of pyrido[2,3-d]pyrimidine-carboxylate derivatives. Indian J. Chem. 2011, 50 (3), 284.

15. Gineinah, M. M.; Nasr, M. N.; Badr, S. M.; El-Husseiny, W. M., Synthesis and antitumor activity of new pyrido[2,3d]pyrimidine derivatives. Med. Chem. Res. 2013, 22 (8), 3943-3952.

16. Hayallah, A. M., Design and synthesis of new 1,8-disubstituted purine-2,6-diones and 3,6-disubstituted thiazolo[2,3-f]purine-2,4-diones as potential antinociceptive and anti-inflammatory agents. pharmacia 2007, 54, 3-13.

17. Bilkei-Gorzo, A.; Abo-Salem, O. M.; Hayallah, A. M.; Michel, K.; Müller, C. E.; Zimmer, A., Adenosine receptor subtype-selective antagonists in inflammation and hyperalgesia. Naunyn-Schmiedeberg's Arch. Pharmacol. 2008, 377 (1), 65-76.

18. Hayallah, A. M.; Isper, S. A.; Al-Okosh, a. A. S., Design and synthesis of some new theophylline linked amides and schiff's bases as bronchodilatros and anti-inflammatory agents. Int. Res. J. Pharm. Pharmacol. 2012, 2 (13), $323-336$.

19. Hafez, H. N.; Abbas, H.-A. S.; El-Gazzar, A.-R. B., Synthesis and evaluation of analgesic, anti-inflammatory and ulcerogenic activities of some triazolo-and 2-pyrazolyl-pyrido[2,3-d]pyrimidines. Acta Pharm. 2008, 58 (4), $359-378$.

20. Said, S.; Abdulla, M., Synthesis and Antiflammatory Activities of Some New Pyridopyridine, Pyridipyrimidine and Pyridopyrimidotriazine Derivatives. World Appl. Sci. J. 2010, 9, 589-599.

21. Fhid, O.; Pawlowski, M.; Filipek, B.; Horodyñska, R.; Maciag, D., Central nervous system activity of new pyrimidine-8-on[2,1-fttheophylline-9-alkylcarboxylic acids derivatives. Pol. J. Pharmacol. 2002, 54 (3), $245-254$.

22. Drabczyńska, A.; Müller, C. E.; Schumacher, B.; Hinz, S.; Karolak-Wojciechowska, J.; Michalak, B.; Pękala, E.; Kieć-Kononowicz, K., Tricyclic oxazolo[2,3-f]purinediones: potency as adenosine receptor ligands and anticonvulsants. Bioorg. Med. Chem. 2004, 12 (18), 4895-4908.

23. Kolaczkowski, M.; Zajdel, P.; Fhid, O.; Duszyñska, B.; Tatarczyñska, E.; Pawlowski, M., Synthesis and 5-HT 1 /A$\mathrm{HT}_{2 \mathrm{~A}}$ activity of some butyl analogues in the group of phenylpiperazine alkyl pyrimido[2,1-f]theophyllines. Pharmacological reports 2005, 57 (229), 229-235.

24. Hayallah, A. M.; Elgaher, W. A.; Salem, O. I.; Alim, A. A. M. A., Design and synthesis of some new theophylline derivatives with bronchodilator and antibacterial activities. Arch. Pharm. Res. 2011, 34 (1), 3-21.

25. Hayallah, A. K.; Talhouni, A. A.; Alim, A. A. M. A., Design and synthesis of new 8-anilide theophylline derivatives as bronchodilators and antibacterial agents. Arch. Pharm. Res. 2012, 35 (8), 1355-1368.

26. Abdel-Aziem, A.; El-Gendy, M. S.; Abdelhamid, A. O., Synthesis and antimicrobial activities of pyrido[2,3d]pyrimidine, pyridotriazolopyrimidine, triazolopyrimidine, and pyrido[2,3-d:6,5-d]dipyrimidine derivatives. Eur. J. Chem. 2012, 3 (4), 455-460.

27. Foley, L. H.; Wang, P.; Dunten, P.; Ramsey, G.; Gubler, M.-L.; Wertheimer, S. J., X-ray Structures of two xanthine inhibitors bound to PEPCK and N-3 modifications of substituted 1,8-Dibenzylxanthines. Bioorg. Med. Chem. Lett. 2003, 13 (21), 3871-3874.

28. Foley, L. H.; Wang, P.; Dunten, P.; Ramsey, G.; Gubler, M.-L.; Wertheimer, S. J., Modified 3-alkyl-1,8dibenzylxanthines as GTP-competitive inhibitors of phosphoenolpyruvate carboxykinase. Bioorg. Med. Chem. Lett. 2003, $13(20), 3607-3610$.

29. Abou-Ghadir, O. F.; Hayallah, A. M.; Abdel-Moty, S. G.; Hussein, M. A., Design and synthesis of some new purine-dione derivatives of potential anti-inflammatory activity. Der Pharma Chemica 2014, 6 (2), 199-211.

30. Skehan, P.; Storeng, R.; Scudiero, D.; Monks, A.; McMahon, J.; Vistica, D.; Warren, J. T.; Bokesch, H.; Kenney, S.; Boyd, M. R., New colorimetric cytotoxicity assay for anticancer-drug screening. J. Natl. Cancer Inst. 1990, 82 (13), 1107-1112.

31. Smellie, A.; Teig, S. L.; Towbin, P., Poling: promoting conformational variation. J. Comput. Chem. 1995, 16 (2), 171-187.

32. Hayallah, A. M.; Famulok, M., Synthesis of new 1,3,8-trisubstitutedpurine-2,6-diones and 1,3,6-trisubstituted thiazolo[2,3-f]purine-2,4-diones. Heterocycles 2007, 74, 369-382.

33. Hayallah, A. M.; Abdel-Hamid, M. K., Design, convenient synthesis and molecular modeling of new pyrido[2,3d]pyrimidine-1,4-dione derivatives as potential anti-inflammatory agents. . Der Pharma Chemica 2014, 6 (5), $45-57$. 
34. Catalyst, 4.11 User Guide, Accelrys Inc., San Diego, CA 92121, USA. 2005.

35. Kurogi, Y.; Guner, O. F., Pharmacophore modeling and three-dimensional database searching for drug design using catalyst. Curr. Med. Chem. 2001, 8 (9), 1035-1055.

36. Li, H.; Sutter, J.; Hoffmann, R., HypoGen: an automated system for generating 3D predictive pharmacophore models. Pharmacophore Perception, Development, and Use in Drug Design 2000, 2, 171.

37. Fischer, P. M.; Lane, D. P., Inhibitors of cyclin-dependent kinases as anti-cancer therapeutics. Curr. Med. Chem. 2000, $7(12), 1213-1245$

38. Drees, M.; Dengler, W. A.; Roth, T.; Labonte, H.; Mayo, J.; Malspeis, L.; Grever, M.; Sausville, E. A.; Fiebig, H. H., Flavopiridol (L86-8275): selective antitumor activity in vitro and activity in vivo for prostate carcinoma cells. Clin. Cancer Res. 1997, 3 (2), 273-279.

39. Goodnow Jr, R. A.; Gillespie, P., 1 Hit and Lead Identification: Efficient Practices for Drug Discovery. Prog. Med. Chem. 2007, 45, 1-62.

40. Zaharevitz, D. W.; Gussio, R.; Leost, M.; Senderowicz, A. M.; Lahusen, T.; Kunick, C.; Meijer, L.; Sausville, E. A., Discovery and initial characterization of the paullones, a novel class of small-molecule inhibitors of cyclin-dependent kinases. Cancer Res. 1999, 59 (11), 2566-2569.

41. Schultz, C.; Link, A.; Leost, M.; Zaharevitz, D. W.; Gussio, R.; Sausville, E. A.; Meijer, L.; Kunick, C., Paullones, a series of cyclin-dependent kinase inhibitors: synthesis, evaluation of CDK1/cyclin B inhibition, and in vitro antitumor activity. J. Med. Chem. 1999, 42 (15), 2909-2919.

42. Meijer, L.; Raymond, E., Roscovitine and other purines as kinase inhibitors. From starfish oocytes to clinical trials. Acc. Chem. Res. 2003, 36 (6), 417-425.

43. Chang, Y.-T.; Gray, N. S.; Rosania, G. R.; Sutherlin, D. P.; Kwon, S.; Norman, T. C.; Sarohia, R.; Leost, M.; Meijer, L.; Schultz, P. G., Synthesis and application of functionally diverse 2, 6, 9-trisubstituted purine libraries as CDK inhibitors. Chem. Biol. 1999, 6 (6), 361-375.

44. Misra, R. N.; Xiao, H.-y.; Kim, K. S.; Lu, S.; Han, W.-C.; Barbosa, S. A.; Hunt, J. T.; Rawlins, D. B.; Shan, W.; Ahmed, S. Z., N-(cycloalkylamino) acyl-2-aminothiazole inhibitors of cyclin-dependent kinase 2. N-[5-[[[5-(1, 1dimethylethyl)-2-oxazolyl] methyl] thio]-2-thiazolyl]-4-piperidinecarboxamide (BMS-387032), a highly efficacious and selective antitumor agent. J. Med. Chem. 2004, 47 (7), 1719-1728.

45. Hoessel, R.; Leclerc, S.; Endicott, J. A.; Nobel, M. E.; Lawrie, A.; Tunnah, P.; Leost, M.; Damiens, E.; Marie, D.; Marko, D., Indirubin, the active constituent of a Chinese antileukaemia medicine, inhibits cyclin-dependent kinases. Nat. Cell Biol. 1999, 1 (1), 60-67.

46. Kuo, G.-H.; DeAngelis, A.; Emanuel, S.; Wang, A.; Zhang, Y.; Connolly, P. J.; Chen, X.; Gruninger, R. H.; Rugg, C.; Fuentes-Pesquera, A., Synthesis and identification of $[1,3,5]$ triazine-pyridine biheteroaryl as a novel series of potent cyclin-dependent kinase inhibitors. J. Med. Chem. 2005, 48 (14), 4535-4546.

47. Bramson, H. N.; Corona, J.; Davis, S. T.; Dickerson, S. H.; Edelstein, M.; Frye, S. V.; Gampe, R. T.; Harris, P. A.; Hassell, A.; Holmes, W. D.; Hunter, R. N.; Lackey, K. E.; Lovejoy, B.; Luzzio, M. J.; Montana, V.; Rocque, W. J.; Rusnak, D.; Shewchuk, L.; Veal, J. M.; Walker, D. H.; Kuyper, L. F., Oxindole-Based Inhibitors of Cyclin-Dependent Kinase 2 (CDK2): Design, Synthesis, Enzymatic Activities, and X-ray Crystallographic Analysis. J. Med. Chem. 2001, 44 (25), 4339-4358.

48. Brana, M. F.; Cacho, M.; García, M. L.; Mayoral, E. P.; López, B.; de Pascual-Teresa, B.; Ramos, A.; Acero, N.; Llinares, F.; Munoz-Mingarro, D., Pyrazolo [3,4-c] pyridazines as novel and selective inhibitors of cyclin-dependent kinases. J. Med. Chem. 2005, 48 (22), 6843-6854.

49. Mettey, Y.; Gompel, M.; Thomas, V.; Garnier, M.; Leost, M.; Ceballos-Picot, I.; Noble, M.; Endicott, J.; Vierfond, J.-m.; Meijer, L., Aloisines, a New Family of CDK/GSK-3 Inhibitors. SAR Study, Crystal Structure in Complex with CDK2, Enzyme Selectivity, and Cellular Effects. J. Med. Chem. 2002, 46 (2), 222-236.

50. $\quad$ Gompel, M.; Leost, M.; De Kier Joffe, E. B.; Puricelli, L.; Franco, L. H.; Palermo, J.; Meijer, L., Meridianins, a new family of protein kinase inhibitors isolated from the Ascidian Aplidium meridianum. Bioorg. Med. Chem. Lett. 2004,14 (7), 1703-1707. 